\title{
Axial vector transition form factors in holographic QCD and their contribution to the anomalous magnetic moment of the muon
}

\author{
Josef Leutgeb $\odot$ and Anton Rebhan 1 \\ Institut für Theoretische Physik, Technische Universität Wien, \\ Wiedner Hauptstrasse 8-10, A-1040 Vienna, Austria
}

(Received 29 December 2019; accepted 1 June 2020; published 17 June 2020)

\begin{abstract}
We evaluate axial vector transition form factors in holographic QCD models that have been shown to reproduce well recent experimental and theoretical results for the pion transition form factor. Comparing with L3 data on $f_{1} \rightarrow \gamma \gamma^{*}$ we find remarkable agreement regarding the shape of single-virtual form factors. In the double-virtual case, the holographic results differ strongly from a simple dipole form, and this has an important impact on the corresponding estimate of the axial vector contribution to the anomalous magnetic moment of the muon $a_{\mu}$ through hadronic light-by-light scattering. We demonstrate that hard-wall models satisfy the Melnikov-Vainshtein short-distance constraint for the latter, if and only if the infinite tower of axial vector states is included. The results for $a_{\mu}$, however, are strongly dominated by the first few resonances. Numerically, these results turn out to be surprisingly large: $(2.9-4.1) \times 10^{-10}$ in the hard-wall models, $57 \%-58 \%$ of which are due to the longitudinal contribution, which is the one responsible for the Melnikov-Vainshtein short-distance constraint. Rescaling the holographic result to obtain an optimal fit of L3 data, but then matching only $52 \%$ of the asymptotic constraint, the result is reduced to $2.2(5) \times 10^{-10}$, which is still significantly larger than most previous phenomenological estimates of the axial vector exchange contribution.
\end{abstract}

DOI: 10.1103/PhysRevD.101.114015

\section{INTRODUCTION AND SUMMARY}

Presently, there is a discrepancy between the measured and the predicted values of the anomalous magnetic moment of the muon [1] of the order of [2-4] $a_{\mu}^{\exp .}$ $a_{\mu}^{\text {theory }} \simeq 26 \times 10^{-10}$, above 3 standard deviations with currently estimated errors. In view of the upcoming new experiment at FERMILAB [5], much effort is being put into reducing the theoretical uncertainty of the Standard Model prediction, which is dominated by hadronic effects [2-4,6-10] (with interesting recent progress in lattice QCD [11-16]), while QED [17] and electroweak effects $[18,19]$ appear under control (see $[1,10]$ for more references).

Although being smaller than the effects of hadronic vacuum polarization, the hadronic light-by-light (HLBL) scattering contribution has a comparable uncertainty. There the exchange of single pseudoscalar mesons $P=\pi^{0}, \eta, \eta^{\prime}$ is the most important contribution. For the latter recent advances have been made in particular using dispersion

Published by the American Physical Society under the terms of the Creative Commons Attribution 4.0 International license. Further distribution of this work must maintain attribution to the author(s) and the published article's title, journal citation, and DOI. Funded by SCOAP ${ }^{3}$. relations $[9,20]$ and lattice QCD [13] to determine the all-important pseudoscalar transition form factors (TFF) $P \rightarrow \gamma^{*} \gamma^{*}$, for which direct experimental information is available almost exclusively in the single-virtual case. However, the HLBL contribution involves both a singlevirtual and a double-virtual TFF (in the external vertex and the internal vertex, respectively).

In Ref. [21], we have recently revisited the predictions of chiral holographic QCD models [22-26]. While these models are certainly only a crude approximation to real QCD (also in the chiral limit), we found that the bottom-up holographic models introduced in [27-29] agree remarkably well with new recent low-energy data [10] for $\pi \rightarrow \gamma \gamma^{*}$ as well as with the results of the dispersive approach for double-virtual pion TFF [9], leading to a result [21] for $a_{\mu}^{\pi^{0}} \simeq 5.9(2) \times 10^{-10}$ which is close to the new evaluations in $[9,10,13]$. The bottom-up holographic models with asymptotic anti-de Sitter (AdS) geometry can be matched to reproduce the leading-order (LO) perturbative $\mathrm{QCD}$ (pQCD) short-distance constraint (SDC) of the vector current two-point function and then even reproduce the exact form of the asymmetry function

$$
\begin{aligned}
f(w) & =\frac{1}{w^{2}}-\frac{1-w^{2}}{2 w^{3}} \ln \frac{1+w}{1-w}, \\
w & =\left(Q_{1}^{2}-Q_{2}^{2}\right) /\left(Q_{1}^{2}+Q_{2}^{2}\right),
\end{aligned}
$$


in the LO pQCD limit of the double-virtual TFF $F\left(Q_{1}^{2}, Q_{2}^{2}\right)$, where $Q_{1,2}^{2}$ are photon virtualities [23].

An important SDC established by Melnikov and Vainshtein (MV) [30] for the four-photon amplitude in the special limit $Q_{1}^{2} \sim Q_{2}^{2} \gg Q_{3}^{2} \rightarrow \infty$ is, however, missed by the pseudoscalar pole contribution to HLBL (unless the single-virtual pion TFF at the external vertex is artificially eliminated and replaced by its on-shell value, a procedure which was proposed in [30] as a simple model to estimate the effect of incorporating the MV-SDC).

Encouraged by the success of bottom-up holographic models in treating the pion-pole contribution, we consider here the axial vector contributions arising from the fivedimensional Chern-Simons action, which is responsible for the correct inclusion of the axial anomaly and which in the chiral holographic models involves one pseudoscalar multiplet and an infinite tower of vector and axial vector mesons. We verify that the latter are indeed responsible for satisfying the MV-SDC, if these complete towers of (axial) vector mesons are included; any truncation leads to a violation at infinite momenta. Nevertheless, in the final result for $a_{\mu}$ only the first few multiplets of axial vector mesons contribute significantly, with the lowest one yielding about $80 \%$ of the complete result.

The TFF of the lightest isoscalar axial vector mesons that are predicted by the holographic models can in fact be compared to experimental data from the L3 Collaboration $[31,32]$. Doing so, we find that the predicted $Q^{2}$ dependence of the single-virtual TFF agrees perfectly with the data, when the parameters of the holographic models are fixed to reproduce $f_{\pi}$ and $m_{\rho}$ (as was done in our study of the pion TFF [21]). With the latter, the hard-wall model of Ref. [29] (called HW2 below) reproduces the MV-SDC parametrically, but numerically only at the level of $62 \%$, while the model of Refs. [27,28] (HW1), which has one more free parameter, can be made to saturate it fully. These models therefore provide a plausible extrapolation of the single-virtual TFF to the double-virtual case needed for evaluating the axial vector contributions to $a_{\mu}$. Using a simple dipole ansatz, Pauk and Vanderhaeghen (PV) [33] have extrapolated the experimental data for the singlevirtual case to estimate $a_{\mu}^{f_{1}}$. The holographic results turn out to have a very different asymptotic behavior and yield much larger contributions. The HW2 model, which reaches $62 \%$ of the asymptotic MV-SDC but agrees well with the low-energy normalization of the axial vector TFF extracted from experiment, yields $a_{\mu}^{\mathrm{AV}} \approx 2.9 \times 10^{-10}$, while the HW1 model, which satisfies $100 \%$ of the MV-SDC but overestimates the low-energy normalization, gives approximately $4.1 \times 10^{-10}$. Approximately $58 \%$ and $57 \%$ of these results $\left(1.7-2.3 \times 10^{-10}\right)$ arise from the longitudinal part of the axial vector meson propagator that is responsible for the MV-SDC. Coincidentally, this is comparable to (albeit smaller than) the extra contribution obtained originally in the $\mathrm{MV}$ model [30], $\Delta a_{\mu}^{\mathrm{PS}, \mathrm{MV}}=$ $2.35 \times 10^{-10}$, where one structure function is artificially kept fixed to its on-shell value. However, when the MV model is updated to current input data [34], this increases to $\Delta a_{\mu}^{\mathrm{PS}, \mathrm{MV}}=3.8 \times 10^{-10}$. Above all, our holographic QCD study (together with our previous evaluation of the pseudoscalar pole contribution [21]) indicates that the simple MV model is not the correct way to implement the MV-SDC, but that additional degrees of freedom are needed for that.

In Ref. [34] a different approach was recently taken to include the MV-SDC by means of an infinite tower of pseudoscalar states (but vehemently criticized in [35]). There the obtained estimate for the effects of the MV-SDC was smaller than our results, $1.3(6) \times 10^{-10}$. As discussed in [34], in the chiral large- $N_{c}$ limit only the lightest pseudoscalar states contribute, while an infinite tower of axial vector mesons is present. The latter were not considered further in [34] on the grounds that they are poorly understood so far and that a good theoretical framework for treating them was missing. The results obtained here demonstrate that they are naturally included in holographic QCD, leading to a somewhat larger estimate of the effects of the MV-SDC than obtained in the model of [34].

This paper is organized as follows. In the next section we briefly recapitulate the holographic hard-wall models already discussed in our previous work on the pion TFF [21], including for comparison also the top-down model of Sakai and Sugimoto, which is found to compare well with low-energy results for the axial vector TFF (in particular the experimental result for its normalization) while missing the SDC. In Sec. III we first compare with the single-virtual results from the L3 Collaboration [31,32]. We then display the axial vector TFF also for the double-virtual case, highlighting its difference from the simple model used in Ref. [33], and work out its asymptotic behavior. In Sec. IV we show that the MV-SDC becomes satisfied when the complete infinite tower of axial vector meson contributions is summed, while each individual contribution decays too fast to do so, and in Sec. V we finally evaluate the contributions to $a_{\mu}$. Since in real QCD, away from the chiral large- $N_{c}$ limit, both excited pseudoscalar mesons and axial vector mesons contribute, we also consider a datadriven adjustment of the holographic results, which are thus used as a mere, albeit sophisticated phenomenological model for the axial vector TFF and the resulting contribution for $a_{\mu}$, and which could be combined with models for excited pseudoscalar mesons along the lines of Ref. [34].

\section{HOLOGRAPHIC QCD MODELS}

The AdS/CFT conjecture [36] has led to many applications in strongly interacting non-Abelian gauge theories in the limit of large color number $N_{c}$. In a top-down 
string-theoretic approach using type-IIA supergravity, Witten [37] has shown that by a supersymmetry-breaking compactification one can construct a model of low-energy QCD based on the near-horizon geometry of D4 branes. Sakai and Sugimoto (SS) $[38,39]$ have extended this model by introducing $N_{f}$ probe D8 and anti-D8 branes localized in the extra dimension of the Witten model, leading to a geometrical realization of chiral symmetry breaking of the $U\left(N_{f}\right)_{\mathrm{L}} \times U\left(N_{f}\right)_{\mathrm{R}}$ symmetry of the unconnected branes, which in the confining geometry are forced to join in the bulk of the higher-dimensional spacetime.

While the SS model is at best a model of low-energy QCD at large $N_{f}$, with no conformal symmetry emerging at high momentum scales, simpler so-called bottom-up models have been constructed that break conformal symmetry by either a hard $[27,28]$ or a soft wall [40] in the bulk. The flavor gauge fields corresponding to chiral symmetry breaking are then introduced by hand and are subjected to appropriate boundary conditions on these walls.

Thus both the top-down and the various bottom-up models eventually describe vector and axial vector mesons through a $U\left(N_{f}\right) \times U\left(N_{f}\right)$ Yang-Mills action in a curved five-dimensional background [with or without a nontrivial dilaton $(\Phi)$ background],

$$
\begin{aligned}
S_{\mathrm{YM}} & \propto \operatorname{tr} \int d^{4} x \int_{0}^{z_{0}} d z e^{-\Phi(z)} \sqrt{-g} g^{P R} g^{Q S} \\
& \times\left(\mathcal{F}_{P Q}^{\mathrm{L}} \mathcal{F}_{R S}^{\mathrm{L}}+\mathcal{F}_{P Q}^{\mathrm{R}} \mathcal{F}_{R S}^{\mathrm{R}}\right),
\end{aligned}
$$

where $P, Q, R, S=0, \ldots, 3, z$ and $\mathcal{F}_{M N}=\partial_{M} \mathcal{B}_{N}-\partial_{N} \mathcal{B}_{M}-$ $i\left[\mathcal{B}_{M}, \mathcal{B}_{N}\right]$.

In the SS model the D8 brane action also involves a Chern-Simons term, which leads to the correct WessZumino-Witten term [38,39]

$$
S_{\mathrm{CS}}=\frac{N_{c}}{24 \pi^{2}} \int \operatorname{tr}\left(\mathcal{B} \mathcal{F}^{2}-\frac{i}{2} \mathcal{B}^{3} \mathcal{F}-\frac{1}{10} \mathcal{B}^{5}\right)
$$

In the bottom-up models, where $\mathcal{B}^{\mathrm{L}}$ and $\mathcal{B}^{\mathrm{R}}$ fields appear separately, the action (3) is added by hand as $S_{\mathrm{CS}}^{\mathrm{L}}-S_{\mathrm{CS}}^{\mathrm{R}}$. The electromagnetic gauge field can be introduced as a nondynamical background field through a nonzero boundary value for the vector gauge field with generator equal to the electric charge matrix, which naturally leads to vector meson dominance (VMD) [39].

As shown in Ref. [41], Eq. (3) implies the correct leading SDC for the structure functions $w_{T, L}\left(Q^{2}\right)$ in the vertex function of two vector and one axial-vector currents, $w_{L}\left(Q^{2}\right)=2 N_{c} / Q^{2}$ (which is exact in the chiral limit), and $w_{T}\left(Q^{2}\right)=N_{c} / Q^{2}$, which does not get perturbative corrections [42], but does receive nonperturbative contributions $[18,43]$ that are suppressed by higher inverse powers of $Q^{2}$, which in holographic QCD depend on the model $[41,44]$.
In the following we recapitulate the relevant formulas for the various models that we will use for deriving the axial vector TFF and their contribution to $a_{\mu}$. For more details see Ref. [21] and references therein.

\section{A. Sakai-Sugimoto model}

With a dimensionless coordinate $Z$ along the connected D8- $\overline{\mathrm{D} 8}$ branes and holographic boundary at $Z= \pm \infty$, the Yang-Mills part of the action of the SS model reads [38,39]

$$
\begin{aligned}
S_{\mathrm{YM}}= & -\kappa \operatorname{tr} \int d^{4} x \int_{-\infty}^{\infty} d Z\left[\frac{1}{2}\left(1+Z^{2}\right)^{-1 / 3} \eta^{\mu \rho} \eta^{\nu \sigma} \mathcal{F}_{\mu \nu} \mathcal{F}_{\rho \sigma}\right. \\
& \left.+\left(1+Z^{2}\right) M_{\mathrm{KK}}^{2} \eta^{\mu \nu} \mathcal{F}_{\mu Z} \mathcal{F}_{\nu Z}\right]
\end{aligned}
$$

with $\kappa=\lambda N_{c} /\left(216 \pi^{3}\right)$ and $\lambda=g_{\mathrm{YM}}^{2} N_{c}$.

An infinite tower of massive vector and axial vector mesons arises from even and odd eigenmodes of $\mathcal{B}_{\mu}^{(n)}=$ $\psi_{n}(Z) v_{\mu}^{(n)}(x)$ with eigenvalue equation

$-\left(1+Z^{2}\right)^{1 / 3} \partial_{Z}\left[\left(1+Z^{2}\right) \partial_{Z} \psi_{n}\right]=\lambda_{n} \psi_{n}, \quad \psi_{n}( \pm \infty)=0$.

The lowest mode $v_{\mu}^{(1)}$ is interpreted as the isotriplet $\rho$ meson [or the $\omega$ meson for the $\mathrm{U}(1)$ generator] with mass $m_{\rho}^{2}=\lambda_{1} M_{\mathrm{KK}}^{2}$. The numerical result $\lambda_{1}=0.669314 \ldots$ fixes the Kaluza-Klein mass of the SS model to $M_{\mathrm{KK}}=$ $1.2223 m_{\rho}$.

The holographic pion mode function is associated with the derivative of the non-normalizable zero mode of (5) of the axial vector sector, $\alpha^{\mathrm{SS}}(Z)=\frac{\pi}{2} \arctan (Z)$. Multiplied with a massless pseudoscalar field in Minkowski space, this appears as the field $\mathcal{B}_{Z}$ or, when the radial gauge $\mathcal{B}_{Z}=0$ is used, in nontrivial boundary conditions on $\mathcal{B}_{\mu}$ [38]. The pion decay constant is given by $f_{\pi}^{2}=$ $\lambda N_{c} M_{\mathrm{KK}}^{2} /\left(54 \pi^{4}\right)$ so that choosing $f_{\pi}=92.4 \mathrm{MeV}$ corresponds to $\kappa=0.00745$ or $\lambda \approx 16.63$ for $N_{c}=3$.

A background electromagnetic field $A_{\mu}(x)$ can be included by setting $\psi( \pm \infty)=1$ for $\mathcal{B}_{\mu}=\mathcal{Q} A_{\mu}(x) \psi(Z)$ with $\mathcal{Q}=e \operatorname{diag}\left(\frac{2}{3},-\frac{1}{3},-\frac{1}{3}\right)$. A real photon with $q^{2}=0$ corresponds to the trivial solution $\psi(Z) \equiv 1$, whereas a virtual photon with spacelike momentum $Q^{2}>0$ is described by a solution where $\lambda_{n} \rightarrow-Q^{2} / M_{\mathrm{KK}}^{2}$. This defines the so-called bulk-to-boundary propagator $\mathcal{J}$, which is determined by

$$
\begin{aligned}
\left(1+Z^{2}\right)^{1 / 3} \partial_{Z}\left[\left(1+Z^{2}\right) \partial_{Z} \mathcal{J}\right] & =\frac{Q^{2}}{M_{\mathrm{KK}}^{2}} \mathcal{J}, \\
\mathcal{J}(Q, Z= \pm \infty) & =1 .
\end{aligned}
$$

At order $1 / N_{c}$ the axial $\mathrm{U}(1)_{A}$ is broken in the SS model, which thereby includes a Witten-Veneziano mechanism 
$[45,46]$ for giving mass to the $\eta_{0}$ pseudoscalar according to $[38,47,48] m_{0}^{2}=N_{f} \lambda^{2} M_{\mathrm{KK}}^{2} /\left(27 \pi^{2} N_{c}\right)$. When explicit mass terms are added $[49,50]$, this indeed gives the right ballpark to account for realistic pseudoscalar meson masses [51].

However, since the SS model is not asymptotically AdS, as it has a diverging dilaton in the UV, it can serve as a holographic model of QCD only at small momenta.

\section{B. Hard-wall models}

In the hard-wall models of Refs. [27-29], the background geometry is instead chosen as pure AdS with metric

$$
d s^{2}=z^{-2}\left(\eta_{\mu \nu} d x^{\mu} d x^{\nu}-d z^{2}\right)
$$

(conformal boundary at $z=0$ ), but with a cutoff at some finite value of the radial coordinate $z_{0}$.

The action for the flavor gauge fields reads

$$
\begin{aligned}
S_{\mathrm{YM}}= & -\frac{1}{4 g_{5}^{2}} \int d^{4} x \int_{0}^{z_{0}} d z \sqrt{-g} g^{P R} g^{Q S} \\
& \times \operatorname{tr}\left(\mathcal{F}_{P Q}^{\mathrm{L}} \mathcal{F}_{R S}^{\mathrm{L}}+\mathcal{F}_{P Q}^{\mathrm{R}} \mathcal{F}_{R S}^{\mathrm{R}}\right),
\end{aligned}
$$

where $P, Q, R, S=0, \ldots, 3, z$ and $\mathcal{F}_{M N}=\partial_{M} \mathcal{B}_{N}-\partial_{N} \mathcal{B}_{M}-$ $i\left[\mathcal{B}_{M}, \mathcal{B}_{N}\right]$.

\section{Hard-wall model with bi-fundamental scalar (HW1)}

In Refs. [27,28], a bifundamental bulk scalar $X$ is introduced, with a five-dimensional mass term determined by the scaling dimension $\Delta=3$ of the chiral-symmetry breaking order parameter $\bar{q} q$ of the boundary theory,

$$
S_{X}=\int d^{4} x \int_{0}^{z_{0}} d z \sqrt{-g} \operatorname{tr}\left(|D X|^{2}+3|X|^{2}\right),
$$

where $D X=\partial X-i \mathcal{B}^{\mathrm{L}} X+i X \mathcal{B}^{\mathrm{R}}$ and $X=U(x, z) v(z) / 2$ with $v(z)=m_{q} z+\sigma z^{3}$, where $m_{q}$ is the quark mass and $\sigma$ the quark condensate.

At a finite value $z_{0}$, a cutoff of $\mathrm{AdS}_{5}$ space is imposed with boundary conditions $\mathcal{F}_{z \mu}^{\mathrm{L}, \mathrm{R}}=0$.

Vector mesons have holographic wave functions given by

$$
\partial_{z}\left[\frac{1}{z} \partial_{z} \psi_{n}(z)\right]+\frac{1}{z} M_{n}^{2} \psi_{n}(z)=0
$$

with boundary conditions $\psi_{n}(0)=\psi_{n}^{\prime}\left(z_{0}\right)=0$, solved by $\psi_{n}(z) \propto z J_{1}\left(M_{n} z\right)$ with $M_{n}$ determined by the zeros of the Bessel function $J_{0}$, denoted by $\gamma_{0, n}$. Identifying $M_{1}=m_{\rho}=775 \mathrm{MeV}$, we obtain

$$
z_{0}=\gamma_{0,1} / m_{\rho}=3.103 \mathrm{GeV}^{-1} .
$$

The vector bulk-to-boundary propagator is obtained by replacing $M_{n}^{2} \rightarrow-Q^{2}$ and choosing the boundary conditions $\mathcal{J}(Q, 0)=1$ and $\partial_{z} \mathcal{J}\left(Q, z_{0}\right)=0$, which gives

$$
\mathcal{J}(Q, z)=Q z\left[K_{1}(Q z)+\frac{K_{0}\left(Q z_{0}\right)}{I_{0}\left(Q z_{0}\right)} I_{1}(Q z)\right] .
$$

The coupling constant $g_{5}$ can be fixed by requiring that the vector current two-point function matches the pQCD result [27]

$$
\Pi_{V}\left(Q^{2}\right)=-\left.\frac{1}{g_{5}^{2} Q^{2}}\left(\frac{1}{z} \partial_{z} \mathcal{J}(Q, z)\right)\right|_{z \rightarrow 0}=-\frac{N_{c}}{24 \pi^{2}} \ln Q^{2},
$$

leading to $g_{5}^{2}=12 \pi^{2} / N_{c}$.

In the chiral limit, the holographic wave functions of the axial vector mesons are given by

$\partial_{z}\left[\frac{1}{z} \partial_{z} \psi_{n}^{A}(z)\right]-g_{5}^{2} \sigma^{2} z^{3} \psi_{n}^{A}(z)+\frac{1}{z}\left(M_{n}^{A}\right)^{2} \psi_{n}^{A}(z)=0$

with the same boundary conditions, $\psi_{n}^{A}(0)=\psi^{A \prime}{ }_{n}\left(z_{0}\right)=0$.

The pion field appears as the longitudinal part of $\mathcal{B}_{M \|}^{A}=\partial_{M} \varphi$. In the chiral limit, its holographic wave function can be given in closed form as $\phi(z)=\mathcal{J}^{A}(0, z)-$ 1 , where $\mathcal{J}^{A}(Q, z)$ is the axial vector bulk-to-boundary propagator with $[22,23]$

$$
\begin{aligned}
\mathcal{J}^{A}(0, z)= & \Psi(z)=\Gamma\left(\frac{2}{3}\right)\left(\xi z^{3} / 2\right)^{1 / 3} \\
& \times\left[I_{-1 / 3}\left(\xi z^{3}\right)-\frac{I_{2 / 3}\left(\xi z_{0}^{3}\right)}{I_{-2 / 3}\left(\xi z_{0}^{3}\right)} I_{1 / 3}\left(\xi z^{3}\right)\right],
\end{aligned}
$$

where $\xi=g_{5} \sigma / 3$. The pion decay constant is determined by [27]

$$
f_{\pi}^{2}=-\left.\frac{1}{g_{5}^{2}}\left(\frac{1}{z} \partial_{z} \Psi(z)\right)\right|_{z \rightarrow 0}
$$

yielding

$$
\frac{6 \pi^{2}}{N_{c}} f_{\pi}^{2}=\frac{\Gamma\left(\frac{2}{3}\right)}{\Gamma\left(\frac{4}{3}\right)} \frac{I_{2 / 3}\left(\xi z_{0}^{3}\right)}{I_{-2 / 3}\left(\xi z_{0}^{3}\right)}(\xi / 2)^{2 / 3}
$$

This fixes $\xi=(0.424 \mathrm{GeV})^{3}$ for $f_{\pi}=92.4 \mathrm{MeV}$.

\section{Hirn-Sanz model (HW2)}

The hard-wall model by Hirn and Sanz [29] (called HW2 in $[21,26])$ does not introduce a matrix-valued scalar field for the purpose of chiral symmetry breaking, but imposes different boundary conditions for vector and axial vector mesons at $z_{0}$, which correspond exactly to the relations that are obtained in the SS model at the point where D8 and anti-D8 branes meet. Vector mesons are given by (10) with $\psi_{n}(0)=\psi_{n}^{\prime}\left(z_{0}\right)=0$ as in the HW1 model, while axial vector mesons satisfy the same eigenvalue equation but with $\psi_{n}^{A}(0)=\psi_{n}^{A}\left(z_{0}\right)=0$ and $\psi^{A \prime}{ }_{n}\left(z_{0}\right) \neq 0$. This gives 
TABLE I. Holographic values of the masses of the three lowest vector mesons in comparison with PDG data for the masses of $\rho$, $\omega$, and $\phi$ mesons in $\mathrm{MeV}$ [52]. All holographic models except HW2(UV-fit) are with $m_{V}=775$ as input; HW2(UV-fit), which is used only for the sake of comparison, is the HW2 model with $g_{5}$ at $N_{c}=3$ matched to $\mathrm{pQCD}$ asymptotics, resulting in a smaller $z_{0}$ and thus much higher $m_{V}$.

\begin{tabular}{llcc}
\hline \hline & \multicolumn{1}{c}{$m_{V}$} & $m_{V^{*}}$ & $m_{V^{* *}}$ \\
\hline SS & 775 & 1606.0 & 2379.3 \\
HW1,2 & 775 & 1778.9 & 2788.8 \\
HW2(UV-fit) & 987.2 & 2266.1 & 3552.6 \\
SW & 775 & 1096.0 & 1342.3 \\
$m_{\rho}$ (PDG) & $775.26(25)$ & $1465(25)$ & $1720(20)$ \\
$m_{\omega}$ (PDG) & $782.65(12)$ & $1425(25)$ & $1670(30)$ \\
$m_{\phi}$ (PDG) & $1019.461(19)$ & $1680(20)$ & \\
\hline \hline
\end{tabular}

$$
\psi_{n}^{A}(z) \propto z J_{1}\left(M_{n}^{A} z\right), \quad M_{n}^{A}=\gamma_{1, n} / z_{0} .
$$

Since $z_{0}$ is already fixed by $m_{\rho}$, this leads to the prediction $M_{1}^{A} / m_{\rho}=\gamma_{1,1} / \gamma_{0,1}=1.593 \cdots$, which is very close to the experimental values $m_{a_{1}(1269)} / m_{\rho} \approx 1.587$ and $m_{f_{1}(1285)} / m_{\omega} \approx$ 1.638. As shown in Tables I and II the results for the lightest axial vector masses in the other models are also close, but not as good, whereas excited (axial) vector masses turn out somewhat too high in all models.

Similar to the SS model, the pion field in the HW2 model is contained in Wilson lines running along the holographic direction, $U(x)=\xi_{\mathrm{R}}(x) \xi_{\mathrm{L}}(x)$ with $\xi_{\mathrm{L}, \mathrm{R}}=$ $P \exp \left(-i \int_{0}^{z_{0}} d z \mathcal{B}_{z}^{\mathrm{L}, \mathrm{R}}\right)$. Its holographic wave function is determined by the axial vector bulk-to-boundary propagator at $Q^{2}=0$,

$$
\mathcal{J}^{A}(0, z)=\Psi(z)=1-\frac{z^{2}}{z_{0}^{2}}
$$

so that (16) yields

$$
g_{5}^{2}=\frac{2}{f_{\pi}^{2} z_{0}^{2}}
$$

Having fixed $z_{0}$ by the $\rho$ meson mass, a realistic choice of $f_{\pi}=92.4 \mathrm{MeV}$ now leads to a coupling $g_{5} \approx 4.932$ which is much smaller than the value $g_{5}=2 \pi \sqrt{N_{c} / 3}$ needed at $N_{c}=3$ to match pQCD according to (13).

In Ref. [21] we have seen that the HW2 model produces a pion TFF that agrees well with existing experimental data when the model is matched to $m_{\rho}$ and $f_{\pi}$. The shortdistance constraints for the pion TFF are then only satisfied at the level of $62 \%$. Setting $g_{5}$ to match the short-distance constraint (13) would lead to a $\rho$ meson mass of $987 \mathrm{MeV}$ and also strong discrepancies with the low-energy pion TFF data which are of crucial importance to the HLBL
TABLE II. Holographic values of the masses of the three lowest axial vector mesons with fixed $m_{\rho}$ in comparison with PDG data for the masses of $a_{1}$ and $f_{1}$ mesons in MeV [52]. (SW1 is the soft-wall version [53] of HW1.)

\begin{tabular}{lccc}
\hline \hline & $m_{A}$ & $m_{A^{*}}$ & $m_{A^{* *}}$ \\
\hline SS & 1186.5 & 2019.8 & 2843.2 \\
HW1 & 1375.5 & 2154.2 & 2995.1 \\
HW2 & 1234.8 & 2260.9 & 3278.6 \\
HW2(UV-fit) & 1573.0 & 2880.1 & 4176.4 \\
SW1 & 1674.1 & 2669.2 & 3497.6 \\
$m_{a_{1}}$ (PDG) & $1230(40)$ & $1655(16)$ & $1930\left(_{-70}^{+30}\right)$ \\
$m_{f_{1}}$ (PDG) & $1281.9(0.5)$ & $1670(30)$ & $1971(15)$ \\
$m_{f_{1}^{\prime}}$ (PDG) & $1426.3(0.9)$ & & \\
\hline \hline
\end{tabular}

contribution to $a_{\mu}$. In the application to $a_{\mu}$ we shall therefore keep the parameters obtained by matching the low-energy regime. This is, however, still an important improvement over the SS model, since the short-distance constraints are then satisfied at least qualitatively. The HW2 model with UV-fitted $g_{5}=2 \pi, N_{c}=3$, and then $z_{0}=$ $2.4359 \mathrm{GeV}^{-1}$ instead of $3.103 \mathrm{GeV}^{-1}$ is referred to as HW2(UV-fit) in Tables I and II.

Because of the extra parameter $\sigma$, the HW1 model is able to incorporate both the desired low-energy parameters $m_{\rho}$ and $f_{\pi}$ as well as the full asymptotic pQCD limits. However, the latter are then presumably reached too quickly for $Q^{2} \gg m_{\rho}^{2}$ because $\mathrm{pQCD}$ corrections typically reduce leading order results by nonnegligible amounts at most energy scales of interest. Taken together we might hope, however, that the HW1 and HW2 models span a plausible range of predictions for real QCD.

In contrast to $[21,26]$, where the pion TFF was studied, we do not include the soft-wall model considered there. The original soft-wall model introduced in [40] is very close to the HW1 model except that it introduces a nontrivial dilaton. As shown in Table II this leads to a poor fit of the mass of the lightest axial vector meson. Moreover the results of Ref. [53] indicate that the HW1 model generally agrees better with pion data than the SW model. In fact, in [21,26], following Ref. [23], a simplified version of the SW model without bi-fundamental bulk scalar $X$ and an ad hoc choice for the pion wave function was used that does not cover the axial vector sector.

\section{AXIAL VECTOR TRANSITION FORM FACTOR}

\section{A. Holographic results}

The effective Lagrangian for the coupling of two photons with one axial vector meson arises from the Chern-Simons action (3) after integrating over the holographic coordinate, which in the case of the SS model reads 


$$
\mathcal{L}_{\mathcal{A} \gamma \gamma}=-i \frac{N_{c}}{12 \pi^{2}} \operatorname{tr} \epsilon^{\mu \nu \rho \sigma} \int_{-\infty}^{\infty} d Z\left(a_{\mu} \mathcal{V}_{\nu}^{\prime} \partial_{\rho} \mathcal{V}_{\sigma}+\mathcal{V}_{\mu} a_{\nu}^{\prime} \partial_{\rho} \mathcal{V}_{\sigma}+\mathcal{V}_{\mu} \mathcal{V}_{\nu}^{\prime} \partial_{\rho} a_{\sigma}\right)
$$

where $a_{\mu}(x, Z)$ is the normalizable axial vector meson field and $\mathcal{V}_{\mu}(x, Z)$ is a vector field whose boundary condition is the background photon field; a prime denotes differentiation with respect to $Z$. With partial integrations this can be simplified to

$$
\mathcal{L}_{\mathcal{A} \gamma \gamma}=-i \frac{N_{c}}{12 \pi^{2}} \operatorname{tr} \epsilon^{\mu \nu \rho \sigma}\left[\int_{-\infty}^{\infty} d Z\left(-3 \mathcal{V}_{\mu}^{\prime} a_{\nu} \partial_{\rho} \mathcal{V}_{\sigma}\right)+\left.\mathcal{V}_{\mu} a_{\nu} \partial_{\rho} \mathcal{V}_{\sigma}\right|_{Z=-\infty} ^{\infty}\right]
$$

In the HW models the integral over $Z$ becomes $2 \int_{0}^{z_{0}} d z(\cdots)$. The boundary term appearing in (22) vanishes in the SS model because $a_{\mu}(x, \pm \infty)=0$. In the HW2 model, the corresponding boundary term also vanishes because $a_{\mu}(x, 0)=0=a_{\mu}\left(x, z_{0}\right)$, but not in the HW1 model, where $a_{\mu}\left(x, z_{0}\right) \neq 0$. Similar to the case of the pion TFF in the HW1 model [23], the resulting nonzero contribution at the infrared wall needs to be subtracted from the Chern-Simons action. Otherwise one would obtain a nonzero amplitude for the decay of an axial vector meson in two real photons (for which $\mathcal{V}_{\mu}$ is simply a constant with respect to the holographic coordinate), and this would violate the Landau-Yang theorem [54]. The latter is realized by the fact that $\mathcal{V}_{\mu}^{\prime}$ in the integral in (22) vanishes when $Q^{2} \rightarrow 0$.

We therefore write the amplitude $\gamma^{*}\left(q_{(1)}\right) \gamma^{*}\left(q_{(2)}\right) \rightarrow \mathcal{A}^{a}$ for photon virtualities $Q_{i}^{2}=-q_{(i)}^{2}$ as

$$
\begin{aligned}
\mathcal{M}^{a}= & i \frac{N_{c}}{4 \pi^{2}} \operatorname{tr}\left(\mathcal{Q}^{2} t^{a}\right) \epsilon_{(1)}^{\mu} \epsilon_{(2)}^{\nu} \epsilon_{\mathcal{A}}^{* \rho} \epsilon_{\mu \nu \rho \sigma} \\
& \times\left[q_{(2)}^{\sigma} Q_{1}^{2} A\left(Q_{1}^{2}, Q_{2}^{2}\right)-q_{(1)}^{\sigma} Q_{2}^{2} A\left(Q_{2}^{2}, Q_{1}^{2}\right)\right],
\end{aligned}
$$

where $\mathcal{Q}=e \operatorname{diag}\left(\frac{2}{3},-\frac{1}{3},-\frac{1}{3}\right)$ and the flavor matrices are given by $t^{a}=\lambda^{a} / 2$ and $t^{0}=1 / \sqrt{6}$. The polarization vectors in (23) are transverse to the respective fourmomenta; writing $\mathcal{M}$ with photon polarization vectors removed, one should supply the corresponding projection operators. The form of (23) is, however, such that no factors $1 / Q_{1,2}^{2}$ are left when doing so:

$$
\begin{aligned}
& \mathcal{M}_{\mu \nu}\left(q_{(1)}, q_{(2)}\right) \\
& \propto \epsilon_{\mathcal{A}}^{* \rho} \epsilon_{\alpha \beta \rho \sigma}\left[\left(q_{(1)}^{2} \delta_{\mu}^{\alpha}-q_{(1)}^{\alpha} q_{(1) \mu}\right) q_{(2)}^{\sigma} \delta_{\nu}^{\beta} A\left(Q_{1}^{2}, Q_{2}^{2}\right)\right. \\
&\left.-\left(q_{(2)}^{2} \delta_{\nu}^{\beta}-q_{(2)}^{\beta} q_{(2) \nu}\right) q_{(1)}^{\sigma} \delta_{\mu}^{\alpha} A\left(Q_{2}^{2}, Q_{1}^{2}\right)\right] .
\end{aligned}
$$

(See Appendix A for the resulting helicity amplitudes.)

In contrast to the model used in Ref. [33], which assumes a similar form of $\mathcal{M}$, the axial vector form factor $A$ following from (22) is not symmetric. ${ }^{1}$ In the HW models, it is given by

\footnotetext{
${ }^{1}$ Even with asymmetric form factor $A$, the form (24) is not the most general one permitted by gauge invariance. The latter admits another independent asymmetric form factor, called $C$ in Ref. [55], which turns out to vanish for the Chern-Simons Lagrangian (22).
}

$$
\begin{aligned}
A\left(Q_{1}^{2}, Q_{2}^{2}\right)= & \frac{2}{Q_{1}^{2}} \int_{0}^{z_{0}} d z\left[\frac{d}{d z} \mathcal{J}\left(Q_{1}, z\right)\right] \mathcal{J}\left(Q_{2}, z\right) \\
& \times \psi^{A}(z) /\left[g_{5}^{-2} \int_{0}^{z_{0}} \frac{d z}{z}\left(\psi^{A}\right)^{2}\right]^{1 / 2}
\end{aligned}
$$

whereas in the SS model we have

$$
\begin{aligned}
A^{\mathrm{SS}}\left(Q_{1}^{2}, Q_{2}^{2}\right)= & \frac{1}{Q_{1}^{2}} \int_{-\infty}^{\infty} d Z\left[\frac{d}{d Z} \mathcal{J}^{\mathrm{SS}}\left(Q_{1}, Z\right)\right] \mathcal{J}^{\mathrm{SS}}\left(Q_{2}, Z\right) \\
& \times \psi_{\mathrm{SS}}^{A}(Z) /\left[\kappa \int_{-\infty}^{\infty} \frac{d Z}{Z}\left(\psi_{\mathrm{SS}}^{A}\right)^{2}\right]^{1 / 2}
\end{aligned}
$$

Because $\frac{d}{d z} \mathcal{J}\left(Q_{1}, z\right)$ vanishes like $Q_{1}^{2}$ in the limit $Q_{1}^{2} \rightarrow 0$, these expressions have a finite limit $A(0,0)$. In the HW models, one obtains

$$
\lim _{Q \rightarrow 0} \frac{1}{Q^{2}} \frac{d}{d z} \mathcal{J}(Q, z)=z \ln \left(z / z_{0}\right)
$$

and in the SS model

$\lim _{Q \rightarrow 0} \frac{M_{\mathrm{KK}}^{2}}{Q^{2}} \frac{d}{d Z} \mathcal{J}^{\mathrm{SS}}(Q, Z)=\frac{Z}{1+Z^{2}}{ }_{2} F_{1}\left(\frac{1}{3}, \frac{1}{2} ; \frac{3}{2} ;-Z^{2}\right)$.

Using this, the result for $A(0,0)$ can be given in closed form for the HW2 model, reading for the lightest axial vector meson

$$
\begin{aligned}
A(0,0) & =-4 \sqrt{2} \frac{J_{0}\left(\gamma_{1,1}\right)-1}{\gamma_{1,1}^{3} J_{0}\left(\gamma_{1,1}\right)} g_{5} z_{0}^{2} \\
& =-0.3502 g_{5} z_{0}^{2}=-16.633 \mathrm{GeV}^{-2}
\end{aligned}
$$

with $g_{5}=\sqrt{2}\left(f_{\pi} z_{0}\right)^{-1} \approx 4.932$. (With UV-fit at fixed $N_{c}$ and $f_{\pi}$, one would obtain $-13.056 \mathrm{GeV}^{-2}$.)

In the HW1 and SS models, the corresponding results have to be obtained numerically. For the former, we find

$$
A(0,0)=-0.3478 g_{5} z_{0}^{2}=-21.043 \mathrm{GeV}^{-2} \quad(\mathrm{HW} 1)
$$


with $g_{5}=\sqrt{12 \pi^{2} / N_{c}}=2 \pi$ and $z_{0}=3.103 \mathrm{GeV}^{-1}$. In the case of the SS model, the result is

$$
A(0,0)=-1.2379 \kappa^{-1 / 2} M_{\mathrm{KK}}^{-2}=-15.926 \mathrm{GeV}^{-2} \quad(\mathrm{SS}) .
$$

\section{B. Comparison with experimental data for $\gamma \gamma^{*} \rightarrow f_{1}$}

The above results for $A(0,0)$ can be compared with experimental data from the L3 Collaboration [31,32] for the so-called equivalent two-photon decay width of the lightest $f_{1}$ mesons with one quasireal longitudinal photon of virtuality $Q_{1}^{2}$ and one real transverse photon [56,57],

$$
\tilde{\Gamma}_{\gamma \gamma}=\lim _{Q_{1}^{2} \rightarrow 0} \Gamma\left(\mathcal{A} \rightarrow \gamma_{L}^{*} \gamma_{T}\right) M_{A}^{2} /\left(2 Q_{1}^{2}\right) .
$$

This is related to the form factor $F_{\mathcal{A} \gamma^{*} \gamma^{*}}^{(1)}$ defined in Ref. [57] by

$$
\tilde{\Gamma}_{\gamma \gamma}=\frac{\pi \alpha^{2} M_{A}}{12}\left[F_{\mathcal{A} \gamma^{*} \gamma^{*}}^{(1)}(0,0)\right]^{2},
$$

which in turn is related to $A(0,0)$ by

$$
M_{A}^{-2} F_{\mathcal{A} \gamma^{*} \gamma^{*}}^{(1)}(0,0)=\frac{N_{c}}{4 \pi^{2}} \operatorname{tr}\left(\mathcal{Q}^{2} t^{a}\right) A(0,0),
$$

where for $f_{1}$ mesons $t^{a}$ can be replaced by a mixture of singlet and octet generators. Similar to $\omega$ and $\phi$, the physical $f_{1}$ and $f_{1}^{\prime}$ states are expected to be close to an ideal mixing scenario, where $f_{1}$ is predominantly $\bar{u} u+\bar{d} d$, while $f_{1}^{\prime}$ is mainly $\bar{s} s$. The experimental results $\tilde{\Gamma}_{\gamma \gamma}=$ $3.5(8) \mathrm{keV}$ for $f_{1}(1285)$ [31] and 3.2(9) $\mathrm{keV}$ for $f_{1}(1420)$ [32], which are fairly close numerically, indicate, however, a certain deviation from ideal mixing; otherwise the radiative decay of $f_{1}^{\prime}$ would be more strongly suppressed compared to $f_{1}$. The mixing angle for the $f_{1}-f_{1}^{\prime}$ system is usually defined as

$$
\left|f_{1}(1285)\right\rangle=\cos \phi_{f}|\bar{n} n\rangle-\sin \phi_{f}|\bar{s} s\rangle
$$

with $|\bar{n} n\rangle=(|\bar{u} u\rangle+|\bar{d} d\rangle) / \sqrt{2}$. Assuming a universal value of $A(0,0)$, the experimental results for $\tilde{\Gamma}_{\gamma \gamma}$ imply $\phi_{f} \approx 20.4^{\circ}$, which is close to the recent LHCb result [58] of $\phi_{f}= \pm(24 \pm 3)^{\circ}$ and other results pointing to a range of $+(20 \ldots 30)^{\circ}[59,60]$. Translated to $A(0,0)$, the experimental results $[31,32]$ thus imply a value of

$$
|A(0,0)|^{\exp .} \simeq 15(2) \mathrm{GeV}^{-2} \text {. }
$$

The above holographic results for the SS and HW2 models agree remarkably well with this, while the HW1 model appears to overestimate the radiative decay amplitudes of the lightest $f_{1}$ mesons by $40 \%$ (corresponding to a factor of 2 for $\tilde{\Gamma}_{\gamma \gamma}$ ).

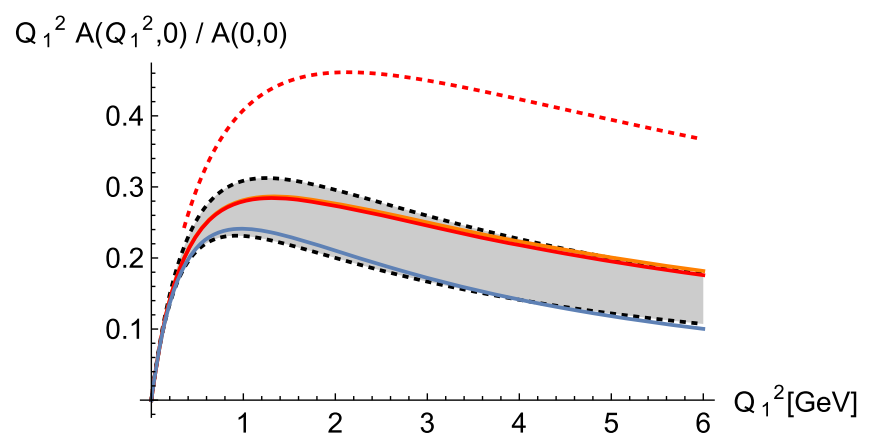

FIG. 1. Single-virtual axial vector TFF from holographic models (SS: blue curve; HW1: orange curve; HW2: red curve) compared with dipole fit of L3 data for $f_{1}(1285)$ (grey band). The parameters of all models are fixed by matching $f_{\pi}$ and $m_{\rho}$. The results for HW1 and HW2 almost coincide, with HW2 at most a line thickness above HW1. When the mass scale $z_{0}^{-1}$ is not fixed by $m_{\rho}$ but matched to the pQCD with $N_{c}=3$, HW2(UV-fit) instead gives the significantly larger result denoted by the red dotted line.

There exist also data on the $Q^{2}$ dependence of $f_{1} \rightarrow \gamma \gamma^{*}$. In the analysis of the L3 data [31,32] the single-virtual TFF of the axial vector mesons has been modeled by a dipole ansatz corresponding to

$$
\frac{A\left(Q_{1}^{2}, 0\right)}{A(0,0)}=\frac{1}{\left(1+Q_{1}^{2} / \Lambda_{D}^{2}\right)^{2}}
$$

with $\Lambda_{D}=1040 \pm 78 \mathrm{MeV}$ and $926 \pm 78 \mathrm{MeV}$ for $f_{1}(1285)$ and $f_{1}(1420)$, respectively.

As will be discussed below, an asymptotic behavior $\sim Q_{1}^{-4}$ is indeed also implied by the holographic HW results, which, however, have a more complicated form at moderate values of $Q_{1}$. In Fig. 1 we compare the experimental fit for $f_{1}(1285)$ to the three holographic results, displaying a remarkable agreement with all of them when their parameters are fixed to match the low-energy input parameters $f_{\pi}$ and $m_{\rho} .{ }^{2}$ (This agreement is, however, spoiled if the HW2 model is forced to exactly match pQCD asymptotically, as also happens in the case of the pion TFF.)

In Fig. 2 we display the holographic results also for the double virtual TFF with $Q_{1}^{2}=Q_{2}^{2}=Q^{2}$. In the calculation of the $f_{1}$ axial vector meson contributions to $a_{\mu}$ by PV [33], the experimental fit has been extrapolated to

$$
\frac{A^{\mathrm{PV}}\left(Q_{1}^{2}, Q_{2}^{2}\right)}{A(0,0)}=\frac{1}{\left(1+Q_{1}^{2} / \Lambda_{D}^{2}\right)^{2}\left(1+Q_{2}^{2} / \Lambda_{D}^{2}\right)^{2}} .
$$

This is represented by the dashed lines in Fig. 2, which deviate rather strongly from the holographic results.

\footnotetext{
${ }^{2}$ The results for $f_{1}(1420)$ are lower than those $f_{1}(1285)$ with some overlap. However, since we consider only chiral models in this paper, a comparison with the result for $f_{1}(1285)$ is more relevant, as the latter is dominantly $\bar{n} n$.
} 


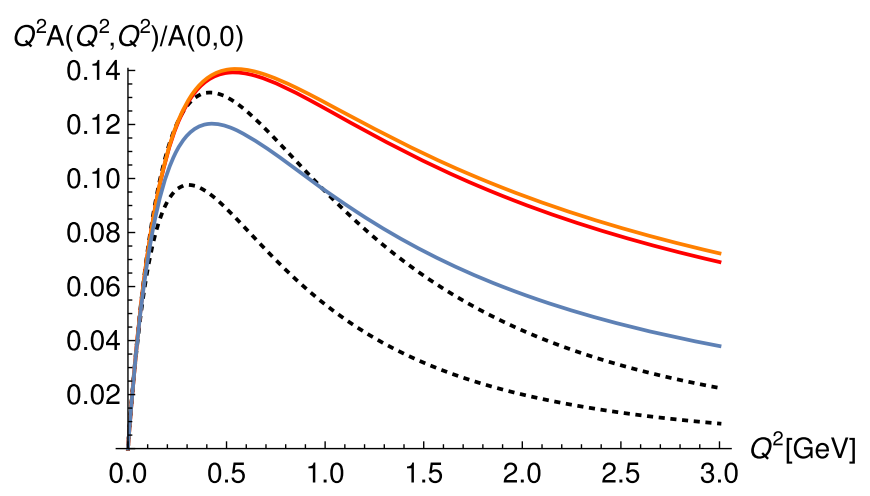

FIG. 2. Double-virtual axial vector TFF for $Q_{1}^{2}=Q_{2}^{2}=Q^{2}$ from holographic models (SS: blue curve; HW1: orange curve; HW2: red curve). The black dashed lines denote the extrapolation of L3 data with a dipole model for each virtuality as used in the calculation of $a_{\mu}^{f_{1}}$ in Ref. [33].

Indeed, the asymptotic behavior of the latter has the same power law in the single and the double virtual cases, as we discuss in the following, while (38) decays like $Q^{-8}$ in the double virtual case.

\section{Asymptotic behavior}

Inspecting the asymptotic behavior in more detail, we introduce the definitions

$$
\begin{aligned}
Q_{1,2}^{2} & =r_{1,2}^{2} Q^{2} \equiv(1 \pm w) Q^{2}, \\
Q^{2} & =\frac{1}{2}\left(Q_{1}^{2}+Q_{2}^{2}\right), \quad w=\left(Q_{1}^{2}-Q_{2}^{2}\right) /\left(Q_{1}^{2}+Q_{2}^{2}\right) .
\end{aligned}
$$

In both HW models we obtain ${ }^{3}$

$$
\begin{aligned}
A\left(Q_{1}^{2}, Q_{2}^{2}\right) \rightarrow & \frac{a A(0,0)}{\left(z_{0} Q\right)^{4}} r_{1} r_{2} \int_{0}^{\infty} d \xi^{3}\left[K_{1}\left(r_{1} \xi\right)\right. \\
& \left.+r_{1} \xi K_{1}^{\prime}\left(r_{1} \xi\right)\right] K_{1}\left(r_{2} \xi\right) \\
= & \frac{a}{\left(z_{0} Q\right)^{4}} \frac{1}{w^{4}}[w(3-2 w) \\
& \left.+\frac{1}{2}(w+3)(1-w) \ln \frac{1-w}{1+w}\right]
\end{aligned}
$$

with a dimensionless constant $a$ which differs between HW1 and HW2. Higher axial vector modes have the same form, but with different $a$. (The SW model has the same asymptotic $Q$ and $w$ dependence.)

The $w$ dependence (displayed in Fig. 3) is asymmetric with a minimum at $w \approx 0.395$ and a logarithmic singularity

\footnotetext{
${ }^{3}$ As we learned through private communication from Martin Hoferichter and now published in [61], the same asymmetry function is obtained when the axial vector TFF is calculated in pQCD with the Brodsky-Lepage formalism.
}

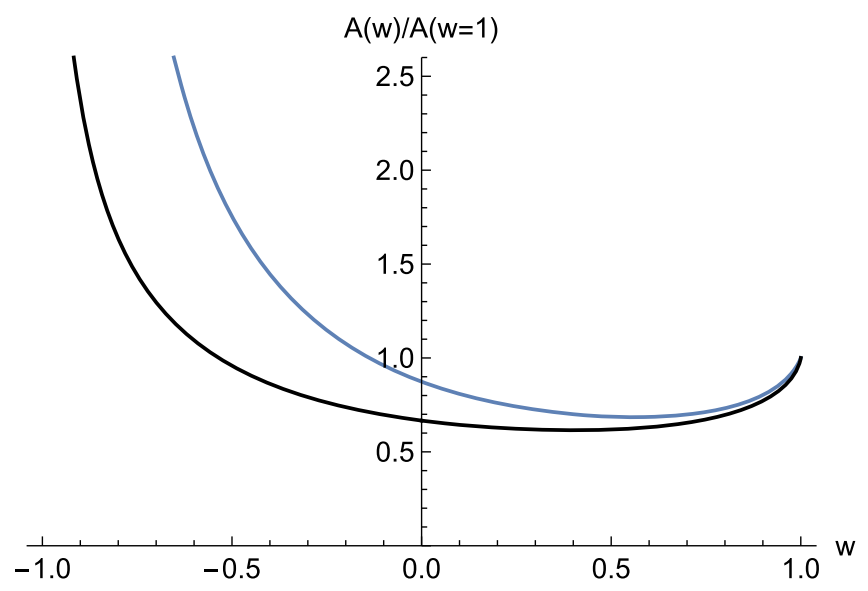

FIG. 3. Dependence of $A\left(Q_{1}^{2}, Q_{2}^{2}\right)$ on the asymmetry parameter $w$ [defined in (39)] in the asymptotically AdS bottom-up models (black line) and in the SS model (blue line). In the single-virtual limit, only $w=+1$ appears in scattering amplitudes.

at $w=-1$, corresponding to a behavior $A \sim \ln \left(Q z_{0}\right) / Q^{4}$ for $A(0, Q \rightarrow \infty)$.

The SS model, which cannot be matched to $\mathrm{pQCD}$ at high momentum scales, decays faster for $Q \rightarrow \infty$, with a qualitatively different $w$ dependence. For completeness, the asymptotic behavior of the form factor $A$ in the SS model is given by

$$
\begin{aligned}
A^{\mathrm{SS}}\left(Q_{1}^{2}, Q_{2}^{2}\right) \rightarrow & \frac{a^{\mathrm{SS}} A^{\mathrm{SS}}(0,0) M_{\mathrm{KK}}^{5}}{Q^{5}} r_{1}^{-1} \\
& \times \int_{0}^{\infty} d \xi \xi^{4}\left(1+3 r_{2}\right) e^{-3\left(r_{1} \xi+r_{2} \xi\right)} \\
= & \frac{a^{\mathrm{SS}} A^{\mathrm{SS}}(0,0) M_{\mathrm{KK}}^{5}}{Q^{5}} \frac{8}{81} \\
& \times \frac{6 \sqrt{1-w}+\sqrt{1+w}}{\sqrt{1+w}(\sqrt{1-w}+\sqrt{1+w})^{6}},
\end{aligned}
$$

and the form of this $w$ dependence is also displayed in Fig. 3. This has its minimum at $w \approx 0.560$ and a power-law singularity at $w=-1$, corresponding to a behavior $A^{\mathrm{SS}} \sim$ $1 / Q^{4}$ for $A^{\mathrm{SS}}(0, Q \rightarrow \infty)$, so that in this particular limit the discrepancy with the bottom-up models is reduced to a merely logarithmic one. Note, however, that the single virtual case $a_{1} / f_{1} \rightarrow \gamma \gamma^{*}$ corresponds to the other limit of $w=+1$.

\section{AXIAL VECTOR CONTRIBUTION TO THE FOUR-PHOTON AMPLITUDE AND LONGITUDINAL SHORT-DISTANCE CONSTRAINTS}

In the Bardeen-Tung-Tarrach basis of the HLBL fourpoint function [20], the short-distance constraint of Melnikov and Vainshtein [30] for $N_{f}=3$ reads [34] 


$$
\lim _{Q_{3} \rightarrow \infty} \lim _{Q \rightarrow \infty} Q^{2} Q_{3}^{2} \bar{\Pi}_{1}\left(Q, Q, Q_{3}\right)=-\frac{2}{3 \pi^{2}}
$$

We shall now show that this constraint is satisfied in the HW models with $g_{5}^{2}=12 \pi^{2} / N_{c}$, which also ensures the correct short-distance limits of single and double virtual pion TFF. In the HW1 model this can be achieved while fitting $m_{\rho}$ to its experimental value, whereas in the HW2 model the latter together with $N_{c}=3$ needs a smaller value of $g_{5}^{2}=2 /\left(f_{\pi} z_{0}\right)^{2}$ so that the SDCs on the pion TFF are satisfied only at the level of $62 \%$ [21].

However, the constraint (42) is only satisfied if the infinite tower of axial vector mesons is taken into account; it is missed completely when only pions and a finite number of axial vector mesons are included.

The axial vector contribution to $\bar{\Pi}_{1}\left(Q, Q, Q_{3}\right)$ comes from the longitudinal part of the axial vector propagator $q_{(3)}^{\mu} q_{(3)}^{\nu} /\left(M_{n}^{A} Q_{3}\right)^{2}$ and has the form

$$
\begin{aligned}
\bar{\Pi}_{1}= & -\frac{g_{5}^{2}}{2 \pi^{4}} \sum_{n=1}^{\infty} \int_{0}^{z_{0}} d z\left[\frac{d}{d z} \mathcal{J}(Q, z)\right] \mathcal{J}(Q, z) \psi_{n}^{A}(z) \frac{1}{\left(M_{n}^{A} Q_{3}\right)^{2}} \\
& \times \int_{0}^{z_{0}} d z^{\prime}\left[\frac{d}{d z^{\prime}} \mathcal{J}\left(Q_{3}, z^{\prime}\right)\right] \psi_{n}^{A}\left(z^{\prime}\right),
\end{aligned}
$$

where we have used that $\sum_{a=0,3,8}\left(N_{c} \operatorname{tr}\left(\mathcal{Q}^{2} t^{a} / e^{2}\right)\right)^{2}=1$. The results of the above section show that at $Q \rightarrow \infty$ the first integral appearing therein behaves as $1 / Q^{2}$ for $Q \rightarrow \infty$, and the second integral, which is a single-virtual form factor, provides a factor of $1 / Q_{3}^{2}$ multiplying a $1 / Q_{3}^{2}$ from the propagator. Thus each summand has a vanishing contribution to (42).

However, the infinite sum behaves differently. This can be demonstrated in closed form in the HW2 model, where both $\mathcal{J}$ and $\psi^{A}$ are given by Bessel functions.

With radial wave functions

$$
\psi_{n}^{A}(z)=\sqrt{2} z z_{0}^{-1} J_{1}\left(\gamma_{1, n} z / z_{0}\right) /\left|J_{0}\left(\gamma_{1, n}\right)\right|
$$

normalized such that $\int_{0}^{z_{0}} d z z^{-1}\left(\psi^{A}(z)\right)_{n}^{2}=1$ one has

$$
\sum_{n=1}^{\infty} \psi_{n}^{A}(z) \psi_{n}^{A}\left(z^{\prime}\right)=z \delta\left(z-z^{\prime}\right)
$$

The sum $\sum_{n=1}^{\infty} \psi_{n}^{A}(z) \psi_{n}^{A}\left(z^{\prime}\right) /\left(M_{n}^{A}\right)^{2}$ is a special case of the axial vector bulk-to-bulk propagator. In a mixed (Euclidean) 4-momentum and radial-coordinate representation the latter is given by

$$
\sum_{n=1}^{\infty} \frac{\psi_{n}^{A}(z) \psi_{n}^{A}\left(z^{\prime}\right)}{Q^{2}+\left(M_{n}^{A}\right)^{2}}=G^{A}\left(Q ; z, z^{\prime}\right)
$$

with

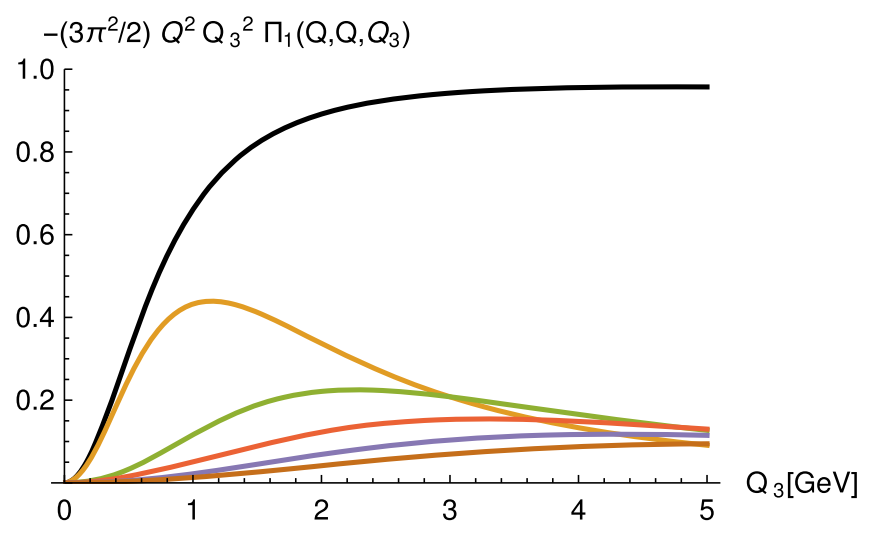

FIG. 4. Axial-vector contribution to $Q_{3}^{2} Q^{2} \bar{\Pi}_{1}\left(Q, Q, Q_{3}\right)$ as a function of $Q_{3}$ at $Q=50 \mathrm{GeV}$ in the HW2 model normalized to the asymptotic value (42) [with prefactor $g_{5}^{2} /(2 \pi)^{2}$ set to one]. The black line corresponds to the infinite sum over the tower of axial vector mesons, and the other lines give the contributions of the first to fifth lightest axial vector mesons.

$$
\begin{aligned}
G^{A}\left(Q ; z, z^{\prime}\right)= & z z^{\prime}\left[K_{1}\left(Q z_{>}\right) I_{1}\left(Q z_{0}\right)\right. \\
& \left.-I_{1}\left(Q z_{>}\right) K_{1}\left(Q z_{0}\right)\right] I_{1}\left(Q z_{<}\right) / I_{1}\left(Q z_{0}\right),
\end{aligned}
$$

where $z_{<}=\min \left(z, z^{\prime}\right)$ and $z_{>}=\max \left(z, z^{\prime}\right)$.

The limiting case of $Q=0$ needed for the longitudinal contribution (43) is given by the simple expression

$$
G^{A}\left(0 ; z, z^{\prime}\right)=\frac{z_{<}^{2}\left(z_{0}^{2}-z_{>}^{2}\right)}{2 z_{0}^{2}} .
$$

Setting $Q z=\xi, \quad Q_{3} z^{\prime}=\xi^{\prime}$, the leading term in $G^{A}\left(0 ; z, z^{\prime}\right)$ at large momenta becomes $z_{<}^{2} / 2=\min \left(\xi^{2} / Q^{2}\right.$, $\left.\xi^{\prime 2} / Q_{3}^{2}\right) / 2$ so that for $Q^{2} \gg Q_{3}^{2}$ we obtain

$$
\begin{aligned}
& -\frac{g_{5}^{2}}{2 \pi^{4}} \frac{1}{2 Q_{3}^{2}} \int_{0}^{\infty} d \xi \int_{0}^{\infty} d \xi^{\prime} \xi K_{1}(\xi) \frac{d}{d \xi}\left[\xi K_{1}(\xi)\right] \\
& \times \frac{d}{d \xi^{\prime}}\left[\xi^{\prime} K_{1}^{\prime}\left(\xi^{\prime}\right)\right] \xi^{2} / Q^{2} \\
& =\frac{g_{5}^{2}}{2 \pi^{4}} \frac{1}{2 Q_{3}^{2}} \int_{0}^{\infty} d \xi \xi K_{1}(\xi) \frac{d}{d \xi}\left[\xi K_{1}(\xi)\right] \xi^{2} / Q^{2} \\
& =-\frac{2}{\pi^{2}} \frac{1}{2 Q_{3}^{2}} \frac{2}{3 Q^{2}}
\end{aligned}
$$

for $g_{5}^{2}=4 \pi^{2}$ at $N_{c}=3$, exactly reproducing the shortdistance constraint (42). Notice that in this limit the singlevirtual form factor effectively gets replaced by $A(0,0)$, something that was done by hand in the model of Ref. [30] to account for the short-distance constraint (albeit in the pseudoscalar sector, whereas here this takes place exclusively in the case of axial vector mesons).

In Fig. 4, the axial vector contribution to $Q_{3}^{2} Q^{2} \bar{\Pi}_{1}(Q$, $\left.Q, Q_{3}\right)$ is plotted for the HW2 model as a function of $Q_{3}$ at $Q=50 \mathrm{GeV}$ for $Q_{3}$ up to $5 \mathrm{GeV}$ so that the kinematic 
regime $Q^{2} \gg Q_{3}^{2} \gg m_{\rho}^{2}$ of the SDC (42) is probed. The full result involving the infinite sum over the tower of axial vector mesons is given by the black line, which is seen to approach the correct limit, while each individual contribution decays for $Q_{3} \rightarrow \infty$.

In the chiral limit, the longitudinal SDC (42) is in fact stronger and holds for all values of $Q_{3}$. Including the pseudoscalar exchange contribution of the HW2 model [21] (but with vanishing pseudoscalar mass) one can readily show that

$$
\lim _{Q \rightarrow \infty} Q^{2} \bar{\Pi}_{1}\left(Q, Q, Q_{3}\right)=-\frac{g_{5}^{2}}{(2 \pi)^{2}} \frac{2}{3 \pi^{2} Q_{3}^{2}}
$$

by partially integrating (43) and using that

$$
\begin{aligned}
\partial_{z^{\prime}} \partial_{z} G^{A}\left(0 ; z, z^{\prime}\right) & =-z \delta\left(z-z^{\prime}\right)-2 z z^{\prime} / z_{0} \\
& =-z \delta\left(z-z^{\prime}\right)-\Psi^{\prime}(z) \Psi^{\prime}\left(z^{\prime}\right) /\left(2 z_{0}^{2}\right),
\end{aligned}
$$

thus verifying that the axial anomaly is correctly implemented. We have checked numerically that this also holds true for the HW1 model, where $\mathcal{J}(Q, z)$ is unchanged but the bulk-to-bulk propagator at zero momentum (48) is replaced by ${ }^{4}$

$$
\begin{aligned}
G^{A}\left(0 ; z, z^{\prime}\right)= & \frac{\pi}{3 \sqrt{3}} z z^{\prime} I_{1 / 3}\left(\xi z_{<}^{3}\right) \\
& \times\left[I_{-1 / 3}\left(\xi z_{>}^{3}\right)-\frac{I_{2 / 3}\left(\xi z_{0}^{3}\right)}{I_{-2 / 3}\left(\xi z_{0}^{3}\right)} I_{1 / 3}\left(\xi z_{>}^{3}\right)\right],
\end{aligned}
$$

which does not obey a relation analogous to (51).

\section{AXIAL VECTOR CONTRIBUTION TO $a_{\mu}$}

Using the method of Gegenbauer polynomials in Ref. [62], the axial vector contribution to the fourphoton amplitude leads to an integral representation of the anomalous magnetic moment of the form (for details see Appendix B)

$$
a_{\mu}^{\mathrm{AV}}=\int_{0}^{\infty} d Q_{1} \int_{0}^{\infty} d Q_{2} \int_{-1}^{1} d \tau \rho_{a}\left(Q_{1}, Q_{2}, \tau\right) .
$$

We have checked our master formula also using the formalism of Refs. [20,63].

In Fig. 5 we compare the integrand obtained with the full tower of axial vector mesons in the HW2 model to the contribution of the first three multiplets at $Q_{1}=Q_{2}$ and $\tau=0$ (implying $Q_{3}=\sqrt{2} Q$ ). This shows that the higher modes, which are essential for satisfying the MV-SDC, contribute only weakly to $a_{\mu}$. In the integrated result

\footnotetext{
${ }^{4}$ However, for $Q \neq 0, G^{A}\left(0 ; z, z^{\prime}\right)$ can no longer be given in closed form but has to be constructed numerically.
}

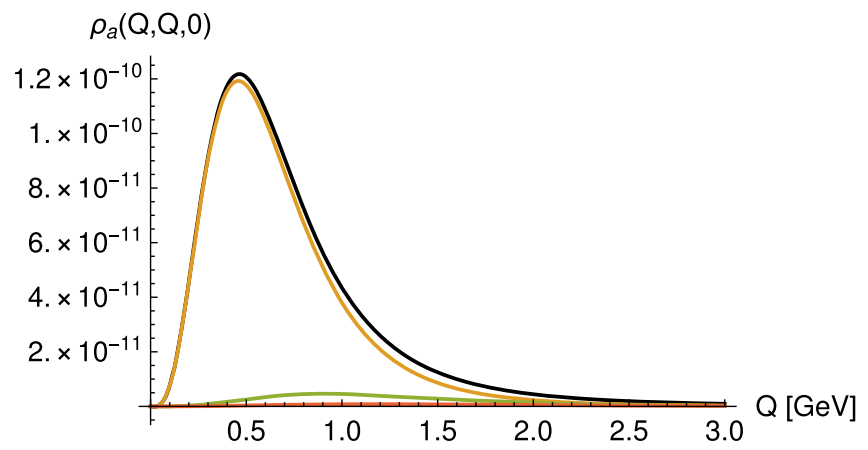

FIG. 5. The integrand $\rho_{a}\left(Q_{1}, Q_{2}, \tau\right)$ in (53) in units of $\mathrm{GeV}^{-2}$ for $Q_{1}=Q_{2}$ and $\tau=0$ (implying $Q_{3}=\sqrt{2} Q$ ) in the case of the HW2 model. The black line is the result from the infinite sum over the tower of axial vector mesons, and the other lines give the contributions of the first to third lightest axial vector meson multiplets.

for $a_{\mu}^{\mathrm{AV}}$, including only the lightest multiplets of axial vectors gives about $80 \%$ of the full result; after that each inclusion of one more multiplet roughly halves the distance to the full result (see Table III). A similar pattern holds for the HW1 model, where we do not have a closed form representation of the infinite tower so that we had to resort to additional numerical estimates. ${ }^{5}$ There the lightest multiplet accounts for about $77 \%$. By contrast, the SS model, which misses the MV-SDC completely and which we will therefore discard in the following, has much smaller contributions from the higher axial vector multiplets.

The numerical results for $a_{\mu}^{\mathrm{AV}}$ in the HW1 and HW2 models, whose parameters have been fixed to reproduce $f_{\pi}$ and $m_{\rho}$, turn out to be surprisingly large, amounting to roughly one-half of the $\pi^{0}$ pole contribution obtained in our previous work [21]. Approximately 58\% of the result $a_{\mu}^{\mathrm{AV}}$ is due to the contribution of the longitudinal part of the axial vector meson propagator, i.e., (1.7-2.4) $\times 10^{-10}$ for the range spanned by HW2 and HW1. This is smaller than the large increase $\left.\Delta a_{\mu}^{\mathrm{PS}}\right|_{\mathrm{MV}}$ obtained in the simple model suggested in Ref. [30] to satisfy the MV-SDC by replacing the external form factor by a constant (which gave $2.35 \times$ $10^{-10}$ in [30], but would be $3.8 \times 10^{-10}$ with current input data [34]), but somewhat higher than the recent estimate of [34] at $\Delta a_{\mu}^{\mathrm{LSDC}}=1.3(6) \times 10^{-10}$. Reference [30] also applied this modification to the transverse part of the axial vector meson contribution, leading to an enhancement by a factor of 2.7 of what their ansatz for the axial vector TFF would otherwise have given, resulting in the estimate

\footnotetext{
${ }^{5}$ With (52) the longitudinal part of the full integrand is given in closed form and therefore can be evaluated directly. The transverse contribution was estimated by evaluation of the lowest seven modes and monitoring the ratio of transverse and longitudinal contributions, from which it was concluded that the accurately determined longitudinal contribution amounts to $57 \%$ of the full result.
} 
TABLE III. The contribution of the infinite tower of axial vector mesons to $a_{\mu}^{\mathrm{AV}}$, calculating in the HW2 model with the analytic expression for the bulk-to-bulk propagator but estimated numerically for the HW1 model (and for comparison also for the SS model, which misses the MV-SDC qualitatively). The entries $j \leq n$ give the contribution of the first $n$ axial vector multiplets. [In the text, HW2(UV-fit) is not considered further because it is a poor fit to IR data, and the SS model is discarded because of its wrong UV behavior.]

\begin{tabular}{lcccccc}
\hline \hline & $j=1$ & $j \leq 2$ & $j \leq 3$ & $j \leq 4$ & $j \leq 5$ & $a_{\mu}^{\mathrm{AV}}$ \\
\hline HW1 & 3.14 & 3.62 & 3.79 & 3.91 & 3.96 & $4.06 \times 10^{-10}$ \\
HW2 & 2.30 & 2.62 & 2.74 & 2.79 & 2.82 & $2.87 \times 10^{-10}$ \\
HW2(UV-fit) & 2.37 & 2.69 & 2.81 & 2.86 & 2.89 & $2.94 \times 10^{-10}$ \\
SS & 1.38 & 1.45 & 1.47 & 1.48 & 1.48 & $1.48 \times 10^{-10}$ \\
\hline \hline
\end{tabular}

$2.2 \times 10^{-10}$ for the lowest axial vector meson multiplet. Our result for the transverse part of the latter amounts instead to $(1.2-1.7) \times 10^{-10}$.

Even when only the lowest multiplet of axial vector mesons is included, we find a substantially larger contribution than is typically estimated in the more recent literature $[1,33]$, where longitudinal and transverse contributions from axial vector mesons are usually not separated. Replacing the holographic form factors by the one used in Ref. [33], Eq. (38), we in fact reproduce the result $0.5 \times$ $10^{-10}$ given therein as a central value for $a_{\mu}\left[f_{1}(1285)\right]$. The main discrepancy arises from the different asymptotic behavior of the form factor, which in Ref. [33] is $Q^{-8}$ in the double virtual case. Another difference is the larger value of $|A(0,0)|$ obtained in the holographic models, which appear to overestimate somewhat the equivalent two-photon decay width $\tilde{\Gamma}_{\gamma \gamma}$. (In the case of the HW2 model, however, the holographic prediction of $4.2 \mathrm{eV}$ for $\tilde{\Gamma}_{\gamma \gamma}$ is completely within the experimental error $(3.5 \pm 0.8 \mathrm{eV})$ when the HW2 model is fitted to correct IR values rather than the pQCD asymptotics.)

If one uses only the holographic results for the normalized form factor $A\left(Q_{1}^{2}, Q_{2}^{2}\right) / A(0,0)$, where the HW1 and HW2 models obtain very similar results, and adjusts the normalization $A(0,0)$ in order to match the experimental result for $\tilde{\Gamma}_{\gamma \gamma}$, we find $a_{\mu}^{f_{1}}=0.88(20) \times 10^{-10}$ for $f_{1}(1285)$, in place of the result $0.5(2) \times 10^{-10}$ given in [33]. Extended to the complete $a_{1}, f_{1}, f_{1}^{\prime}$ multiplet, this leads to $a_{\mu}^{\mathrm{AV} 1}=1.74(40) \times 10^{-10}$, which can be viewed as a data-driven result, where holographic QCD is used as an interpolator from single-virtual data to the double-virtual domain. $^{6}$

The full axial vector exchange contribution will also involve higher multiplets, for which at present no data are

\footnotetext{
${ }^{6}$ For future tests of the latter, we have included in Appendix A the expressions for the helicity amplitudes for photoproduction of axial vector mesons.
}

available. Assuming that the lowest multiplet again accounts for $80 \%$ of the total contribution, our estimate for the latter is $a_{\mu}^{\mathrm{AV}}=2.2(5) \times 10^{-10}$.

This downscaling of our holographic results to match experimental data implies that the MV-SDC is satisfied to a lesser degree (unless it is applied only to a finite number of axial vector multiplets). The HW2 model with IR-fixed parameters, which reaches only $62 \%$ of the MV-SDC, needs only a moderate change, while the HW1 model, which saturates the MV-SDC, requires a much stronger one. After such an overall downscaling, both HW models have almost exactly the same level of $52 \%$ to which the MV-SDC is met. This could perhaps be interpreted as an indication that in models that are closer to real QCD other contributions may be of comparable importance, notably from the excited pseudoscalar mesons [34], which in the chiral large- $N_{c}$ limit of our holographic models do not arise.

\section{CONCLUSION}

In the present study, we have calculated the axial vector meson contributions arising from the Chern-Simons action in the holographic QCD models, for which we had reevaluated the pion-pole contribution to the anomalous magnetic moment of the muon in Ref. [21]. We found that the infinite tower of axial vector mesons present in all these models leads to a large-momentum behavior that in the case of hard-wall models matches the MV-SDC [30] concurrently with the SDC for the pion TFF.

At low energies, we found that the holographic QCD models, now including the top-down SS model for lowenergy large- $N_{c}$ QCD, reproduce well the experimentally determined shape of the $f_{1}(1285)$ TFF. The SS and HW2 models also agree with its normalization, which is related to $\tilde{\Gamma}_{\gamma \gamma}$, while the HW1 model overestimates the latter. On the other hand, the HW2 model saturates the MV-SDC only at the level of $62 \%$, whereas the HW1 model does so completely.

In Ref. [21] we have found that the HW1 and HW2 models bracket the low-energy results for the pion TFF, giving also the high and low ends of our results for $a_{\mu}^{\pi^{0}}=5.9(2) \times 10^{-10}$. Evaluating the axial vector contributions we obtained $a_{\mu}^{\mathrm{AV}}=4.06 \times 10^{-10}$ and $2.87 \times 10^{-10}$, respectively, $57 \%$ and $58 \%$ of which are coming from the longitudinal contribution responsible for the MV-SDC. This is somewhat larger than the estimate of $\Delta a_{\mu}^{\mathrm{LSDC}}$ obtained recently in [34].

The HW1 result, which gives a similarly high result while completely saturating the MV-SDC, appears to significantly overestimate the measured two-photon rate of $f_{1}(1285)$, so that one might favor the smaller result coming from the HW2 model. On the other hand, real QCD has excited axial vector mesons that are lighter than predicted by the holographic models (see Table II), and 
TABLE IV. Summary of the results of our previous calculation of the pseudoscalar pole contribution of Ref. [21] and the results obtained here for the contribution of the infinite tower of axial vector mesons in the holographic models HW1 and HW2, where the last column (Extrapolation) contains the span of the (ground-state) pseudoscalar pole contributions in the two models, and the rescaled result for the axial vector contributions to match L3 data for $f_{1}(1285)$ and $f_{1}(1420)$.

\begin{tabular}{lccc}
\hline \hline & HW1 & HW2 & Extrapolation \\
\hline$a_{\mu}^{\mathrm{PS}}\left[\pi^{0}+\eta+\eta^{\prime}\right] \times 10^{10}$ & $9.22[6.13+1.67+1.42]$ & $8.37[5.92+1.59+1.34]$ & $8.8(4)$ \\
$a_{\mu}^{\mathrm{AV}}[L+T] \times 10^{10}$ & $4.06[2.32+1.74]$ & $2.87[1.66+1.20]$ & $2.2(5)[1.3(3)+0.9(2)]$ \\
$a_{\mu}^{\mathrm{PS}+\mathrm{AV}} \times 10^{10}$ & 13.3 & 11.2 & $11.0(6)$ \\
\hline \hline
\end{tabular}

those will also contribute to $a_{\mu}^{\mathrm{AV}}$. Moreover, away from the chiral limit excited pseudoscalar mesons have to be included, as pointed out in [34]. This could mean that the final result for $\Delta a_{\mu}$ from the pseudoscalar and axial vector sector might indeed be in between the HW1 and HW2 results.

We have also considered the possibility of using the holographic results for the axial vector TFF as a phenomenological interpolator, where the normalization is fitted to the experimental results for $\gamma \gamma^{*} \rightarrow f_{1}$. Consistency of the results for $f_{1}(1285)$ and $f_{1}(1420)$ leads to a mixing angle $\phi_{f} \approx 20^{\circ}$ away from ideal mixing, in agreement with other phenomenological studies. Using the resulting overall normalization, we arrived at the result $a_{\mu}^{\mathrm{AV} 1}=1.74(40) \times$ $10^{-10}$ for the lightest axial vector meson multiplet. This is also significantly larger than was obtained in previous recent studies using various ansätze for the TFF [1,33], which $\operatorname{gave}^{7} a_{\mu}^{\mathrm{AV} 1}=(0.4-1) \times 10^{-10}$. Since our study suggests that $a_{\mu}^{\mathrm{AV} 1}$ may account for only $80 \%$ of the axial vector meson sector (perhaps even less, since the holographic models overestimate the mass of excited axial vector mesons), also this more data-based approach suggests a contribution of (at least) $a_{\mu}^{\mathrm{AV}}=2.2(5) \times 10^{-10}$, close to the pristine result of the HW2 model.

In summary, our holographic results underline the numerical importance of axial vector contributions, and their role in satisfying the MV-SDC [30]. However, our results for the effect of the latter, which can be attributed to $57 \%-58 \%$ of the axial vector contribution, are significantly smaller than what is obtained by the simple MV model (when updated to modern input data), where in the pseudoscalar contributions one structure function is artificially kept fixed to its on-shell value. More importantly, the holographic QCD calculation indicates that the MV model is not the correct way to implement the MV-SDC, but that additional degrees of freedom are needed. On the other hand, our results are larger than (albeit not too far above) the estimate in [34] from a model involving an infinite tower of pseudoscalar excitations. Moreover, the axial vector TFF obtained in holographic QCD provide a well motivated model for the double-virtual case which differs

\footnotetext{
${ }^{7}$ Two very recent papers $[55,64]$ arrived at even lower results.
}

strongly from a simple dipole ansatz, suggesting that previous estimates of the axial vector contribution to $a_{\mu}$ are significantly too small.

After completion of this work, Ref. [65] appeared, which also has worked out the contribution of axial vector mesons in the HW2 model, in essence agreeing with our findings, but employing different sets of parameters (with decay constants chosen differently for a partition in $\pi^{0} / a_{1}, \eta / f_{1}$, and $\eta^{\prime} / f_{1}^{\prime}$ sectors). ${ }^{8}$ Their choice "Set 1 " corresponds roughly to our treatment of the HW2 model, where a fit of $f_{\pi}$ and $m_{\rho}$ implies that only $62 \%$ of the MV SDC is reached; "Set 2" corresponds to what we called HW2(UVfit), where $100 \%$ of the MV SDC is satisfied at the expense of a much too large $\rho$ meson mass, whereas we have employed the HW1 model to be able to match SDCs as well as the low-energy parameters. Since Ref. [65] included the pseudoscalar contributions in their final results, let us point out that our results are to be added to our previous results of the pseudoscalar pole contribution calculated before in Ref. [21]. To facilitate a comparison of our results with those of Ref. [65], our final results for the pseudoscalar plus axial vector sector are rendered in Table IV, where the last column corresponds to the adjustment of the axial vector contribution to match L3 data. As stated above, this downscaled result could be viewed as an extrapolation to real QCD, where in contrast to the chiral large- $N_{c}$ limit excited pseudoscalars are also contributing and which one would then have to add in [34].

\section{ACKNOWLEDGMENTS}

We are indebted to Massimiliano Procura and Jan Lüdtke for most useful discussions and suggestions, and for help with the results of Refs. [20,63]. We cordially thank the authors of Ref. [65] for correspondence after the

\footnotetext{
${ }^{8}$ Treating $f_{1}$ and $f_{1}^{\prime}$ in line with $\eta$ and $\eta^{\prime}$, the authors of Ref. [65] found the $f_{1}^{\prime}$ axial vector mesons to give the largest contribution, whereas both ideal mixing and the mixing with $\phi_{f}=20.4^{\circ}$ we inferred from experimental data should have the largest contribution coming from $f_{1}$, in particular given the higher mass of $f_{1}^{\prime}$. In our models, where the axial vector meson multiplets have strictly degenerate masses as given in Table II, mixing with $\phi_{f}=20.4^{\circ}$ implies a partition of $25 \%, 49 \%, 26 \%$ for the $a_{1}, f_{1}, f_{1}^{\prime}$ contributions, whereas in real QCD the $f_{1}^{\prime}$ contribution should be somewhat reduced by its higher mass.
} 
publication of our respective works, and also Hans Bijnens, Gilberto Colangelo, Franziska Hagelstein, and Martin Hoferichter for comments on the manuscript. J.L. was supported by the FWF doctoral program Particles \& Interactions, Project No. W1252-N27.

\section{APPENDIX A: HELICITY AMPLITUDES FOR $\gamma^{*} \gamma^{*} \rightarrow \mathcal{A}$}

For future potential tests of the holographic predictions in the double-virtual case, we list here the helicity amplitudes for photoproduction of axial vector mesons, generalizing the formulas given in Eq. (C21) of Ref. [57] from a symmetric structure function $A\left(Q_{1}^{2}, Q_{2}^{2}\right)$ to the asymmetric one appearing in (25). Note that the generic form of the amplitude admits one further structure function [denoted by $C\left(Q_{1}^{2}, Q_{2}^{2}\right)$ in Ref. [55]], which vanishes in the holographic result (23).

With the definitions [57]

$$
\begin{aligned}
\nu & :=q_{(1)} \cdot q_{(2)}=\frac{1}{2}\left(M_{A}^{2}+Q_{1}^{2}+Q_{2}^{2}\right), \\
X & :=\nu^{2}-Q_{1}^{2} Q_{2}^{2},
\end{aligned}
$$

and the overall constant $C:=\operatorname{tr}\left(\mathcal{Q}^{2} t^{a}\right) N_{c} /\left(4 \pi^{2}\right)$, the holographic result for the amplitude (23) in terms of the functions $A \equiv A\left(Q_{1}^{2}, Q_{2}^{2}\right)$ and $\bar{A} \equiv A\left(Q_{2}^{2}, Q_{1}^{2}\right)$ contains the following nonzero $\gamma^{*} \gamma^{*} \rightarrow \mathcal{A}$ helicity amplitudes:

$$
\begin{aligned}
-i \mathcal{M}_{++} / C= & \frac{\nu}{M_{A}}\left(Q_{1}^{2} A-Q_{2}^{2} \bar{A}\right)-\frac{Q_{1}^{2} Q_{2}^{2}}{M_{A}}(A-\bar{A}), \\
& -i \mathcal{M}_{0+} / C=Q_{1}\left(\nu A+Q_{2}^{2} \bar{A}\right), \\
& -i \mathcal{M}_{-0} / C=Q_{2}\left(\nu \bar{A}+Q_{1}^{2} A\right),
\end{aligned}
$$

where the first two indices refer to the helicities of the two virtual photons.

The structure functions $F_{\mathcal{A \gamma ^ { * } \gamma ^ { * }}}^{(0)}\left(Q_{1}^{2}, Q_{2}^{2}\right)$ and $F_{\mathcal{A \gamma ^ { * } \gamma ^ { * }}}^{(1)}\left(Q_{1}^{2}\right.$, $Q_{2}^{2}$ ) defined in Eq. (C14) of Ref. [57] are proportional to $\mathcal{M}_{++} /\left[\left(Q_{1}^{2}-Q_{2}^{2}\right) \nu / M_{A}^{3}\right]$ and $\mathcal{M}_{0+} /\left[Q_{1} X /\left(\nu M_{A}^{2}\right)\right]$, respectively.

\section{APPENDIX B: INTEGRAL REPRESENTATION OF $a_{\mu}^{\mathrm{AV}}$}

With the method of Gegenbauer polynomials described in Ref. [62] we have obtained

$$
a_{\mu}^{\mathrm{AV}}=-\frac{2 \alpha^{3}}{3 \pi^{2}} \int_{0}^{\infty} d Q_{1} d Q_{2} \int_{-1}^{+1} d \tau \sqrt{1-\tau^{2}} Q_{1}^{3} Q_{2}^{3}\left(K_{1}+K_{2}\right),
$$

with $K_{1}$ the integral kernel for the $s$-channel reading

$$
\begin{aligned}
K_{1}= & \frac{A\left(Q_{3}^{2}, 0\right)\left(Q_{1}^{2} A\left(Q_{1}^{2}, Q_{2}^{2}\right)+Q_{2}^{2} A\left(Q_{2}^{2}, Q_{1}^{2}\right)\right)}{2 Q_{1} Q_{2} Q_{3}^{2} m_{\mu}^{2} M_{A}^{2}}\left[\tau\left(Q_{2}^{2}\left(4 \sigma_{2}^{E}+\left(\sigma_{2}^{E}\right)^{2}-5\right)-8 m_{\mu}^{2}\right)\right. \\
& \left.-4 Q_{2} Q_{1}\left(-4\left(\tau^{2}-1\right) X m_{\mu}^{2}+2 Q_{2}^{2} X-\sigma_{1}^{E}-\sigma_{2}^{E}+2\right)-8 Q_{2} Q_{1}^{3} X+Q_{1}^{2} \tau\left(-16 Q_{2}^{2} X+4 \sigma_{1}^{E}+\left(\sigma_{1}^{E}\right)^{2}-5\right)\right] \\
& +\frac{A\left(Q_{1}^{2}, Q_{2}^{2}\right) A\left(Q_{3}^{2}, 0\right)}{2 Q_{1} Q_{2}^{2} Q_{3}^{2} m_{\mu}^{2}\left(M_{A}^{2}+Q_{3}^{2}\right)}\left[Q_{2}^{3} Q_{1}^{2} \tau\left(-8 Q_{2}^{2} X+2 \sigma_{1}^{E}+\left(\sigma_{1}^{E}\right)^{2}-2 \sigma_{2}^{E} \tau^{2}+8 \sigma_{2}^{E}+\left(\sigma_{2}^{E}\right)^{2} \tau^{2}+\left(\sigma_{2}^{E}\right)^{2}+\tau^{2}-12\right)\right. \\
& -4 m_{\mu}^{2}\left(Q_{2}^{3} \tau+Q_{1}^{3}\left(1-4 Q_{2}^{2} \tau^{2} X\right)+Q_{2}^{2} Q_{1}\left(4 Q_{2}^{2} X+\tau^{2}\right)-4 Q_{2} Q_{1}^{4} \tau X+Q_{2} Q_{1}^{2} \tau\left(4 Q_{2}^{2} X+3\right)\right) \\
& +2 Q_{2}^{4} Q_{1}\left(-2 Q_{2}^{2} X+\sigma_{1}^{E}-\sigma_{2}^{E} \tau^{2}+2 \sigma_{2}^{E}+\left(\sigma_{2}^{E}\right)^{2} \tau^{2}-3\right)+2 Q_{2}^{2} Q_{1}^{3}\left(-6 Q_{2}^{2} X+\sigma_{1}^{E}+2 \sigma_{2}^{E}-3\right) \\
& \left.-4 Q_{2} Q_{1}^{4} \tau\left(4 Q_{2}^{2} X-\sigma_{1}^{E}+1\right)+Q_{1}^{5}\left(-8 Q_{2}^{2} X+2 \sigma_{1}^{E}-2\right)+Q_{2}^{5}\left(\left(\sigma_{2}^{E}\right)^{2}-1\right) \tau\right] \\
& +\frac{A\left(Q_{2}^{2}, Q_{1}^{2}\right) A\left(Q_{3}^{2}, 0\right)}{2 Q_{1}^{2} Q_{2} Q_{3}^{2} m_{\mu}^{2}\left(M_{A}^{2}+Q_{3}^{2}\right)}\left[2 Q_{1}^{2}\left(-2 Q_{2} \tau^{2} m_{\mu}^{2}+Q_{2}^{3}\left(8 \tau^{2} X m_{\mu}^{2}+2 \sigma_{1}^{E}+\sigma_{2}^{E}-3\right)-4 Q_{2}^{5} X\right)-4 Q_{2} Q_{1}^{6} X\right. \\
& -2 Q_{2} Q_{1}^{4}\left(8 X m_{\mu}^{2}+6 Q_{2}^{2} X+\sigma_{1}^{E} \tau^{2}-2 \sigma_{1}^{E}-\left(\sigma_{1}^{E}\right)^{2} \tau^{2}-\sigma_{2}^{E}+3\right) \\
& \times Q_{1}^{3} \tau\left(Q_{2}^{2}\left(-16 Q_{2}^{2} X-2 \sigma_{1}^{E}\left(\tau^{2}-4\right)+\left(\sigma_{1}^{E}\right)^{2}\left(\tau^{2}+1\right)+2 \sigma_{2}^{E}+\left(\sigma_{2}^{E}\right)^{2}+\tau^{2}-12\right)-4 m_{\mu}^{2}\left(4 Q_{2}^{2} X+1\right)\right) \\
& \left.-2 Q_{2}^{3}\left(2 m_{\mu}^{2}-Q_{2}^{2}\left(\sigma_{2}^{E}-1\right)\right)+4 Q_{2}^{2} Q_{1} \tau\left(m_{\mu}^{2}\left(4 Q_{2}^{2} X-3\right)+Q_{2}^{2}\left(\sigma_{2}^{E}-1\right)\right)-Q_{1}^{5} \tau\left(8 Q_{2}^{2} X-\left(\sigma_{1}^{E}\right)^{2}+1\right)\right],
\end{aligned}
$$

and $K_{2}$ for the $t$ - and $u$-channels, 


$$
\begin{aligned}
K_{2}= & \frac{A\left(Q_{2}^{2}, 0\right)\left(Q_{3}^{2} A\left(Q_{3}^{2}, Q_{1}^{2}\right)+Q_{1}^{2} A\left(Q_{1}^{2}, Q_{3}^{2}\right)\right)}{Q_{1} Q_{2} Q_{3}^{2} m_{\mu}^{2} M_{A}^{2}}\left(-4 \tau m_{\mu}^{2}-4 Q_{1} Q_{2}\left(\tau^{2}-1\right)\left(-4 X m_{\mu}^{2}+2 Q_{2}^{2} X-\sigma_{1}^{E}+1\right)+Q_{1}^{2}\left(\left(\sigma_{1}^{E}\right)^{2}-1\right) \tau\right) \\
& +\frac{A\left(Q_{1}^{2}, Q_{3}^{2}\right) A\left(Q_{2}^{2}, 0\right)}{Q_{1} Q_{2} Q_{3}^{2} m_{\mu}^{2}\left(M_{A}^{2}+Q_{2}^{2}\right)}\left[2 Q_{2} Q_{1}^{3}\left(4 Q_{2}^{2}\left(\tau^{2}+1\right) X-4 \sigma_{1}^{E} \tau^{2}+\left(\sigma_{1}^{E}\right)^{2} \tau^{2}-\sigma_{2}^{E}+3 \tau^{2}+1\right)\right. \\
& +Q_{2}^{2} Q_{1}^{2} \tau\left(-2 \sigma_{1}^{E}+\left(\sigma_{1}^{E}\right)^{2}-6 \sigma_{2}^{E}-\left(\sigma_{2}^{E}\right)^{2}+8\right)+Q_{1}^{4} \tau\left(16 Q_{2}^{2} X-6 \sigma_{1}^{E}+\left(\sigma_{1}^{E}\right)^{2}+5\right) \\
& -4 m_{\mu}^{2}\left(Q_{2}^{2} \tau+4 Q_{2} Q_{1}^{3}\left(\tau^{2}+1\right) X+2 Q_{1}^{2} \tau\left(6 Q_{2}^{2} X-1\right)+Q_{2} Q_{1}\left(4 Q_{2}^{2} X-1\right)\right) \\
& \left.+2 Q_{2}^{3} Q_{1}\left(-2 Q_{2}^{2} X+\sigma_{1}^{E}-3 \sigma_{2}^{E}+2\right)+Q_{2}^{4}\left(\left(\sigma_{2}^{E}\right)^{2}-1\right) \tau+4 Q_{2} Q_{1}^{5} X\right] \\
& -\frac{A\left(Q_{2}^{2}, 0\right) A\left(Q_{3}^{2}, Q_{1}^{2}\right)}{Q_{1}^{2} Q_{2} Q_{3}^{2} m_{\mu}^{2}\left(M_{A}^{2}+Q_{2}^{2}\right)}\left[10 Q_{2}^{4} Q_{1}\left(\sigma_{2}^{E}-1\right) \tau+2 Q_{2}^{5}\left(\sigma_{2}^{E}-1\right)-4 Q_{2} Q_{1}^{6} X\right. \\
& +4 m_{\mu}^{2}\left\{4 Q_{2} Q_{1}^{4}\left(\tau^{2}+1\right) X+2 Q_{1}^{3} \tau\left(2 Q_{2}^{2}\left(2 \tau^{2}+3\right) X-1\right)\right. \\
& \left.+Q_{2} Q_{1}^{2}\left(4 Q_{2}^{2}\left(3 \tau^{2}+1\right) X-4 \tau^{2}-1\right)+2 Q_{2}^{2} Q_{1} \tau\left(2 Q_{2}^{2} X-1\right)-Q_{2}^{3}\right\} \\
& -Q_{2}^{2} Q_{1}^{3} \tau\left(16 Q_{2}^{2}\left(\tau^{2}+1\right) X-2 \sigma_{1}^{E}\left(5 \tau^{2}+2\right)+\left(\sigma_{1}^{E}\right)^{2}\left(\tau^{2}+1\right)-10 \sigma_{2}^{E}-\left(\sigma_{2}^{E}\right)^{2}+9 \tau^{2}+14\right) \\
& -Q_{1}^{5} \tau\left(24 Q_{2}^{2} X-6 \sigma_{1}^{E}+\left(\sigma_{1}^{E}\right)^{2}+5\right)+2 Q_{2}^{3} Q_{1}^{2}\left(\tau^{2}\left(-4 Q_{2}^{2} X+2 \sigma_{1}^{E}-9\right)+\sigma_{2}^{E}\left(6 \tau^{2}+2\right)+\left(\sigma_{2}^{E}\right)^{2} \tau^{2}-2\right) \\
& \left.-2 Q_{2} Q_{1}^{4}\left(2 Q_{2}^{2}\left(10 \tau^{2}+1\right) X-8 \sigma_{1}^{E} \tau^{2}+\left(\sigma_{1}^{E}\right)^{2} \tau^{2}-\sigma_{2}^{E}+7 \tau^{2}+1\right)\right],
\end{aligned}
$$

for one axial vector meson multiplet with mass $M_{A}$ and form factors as defined in (25), where we have used the notation of $[20,63]$,

$$
\begin{aligned}
Q_{3}^{2} & =Q_{1}^{2}+2 Q_{1} Q_{2} \tau+Q_{2}^{2}, \quad X=\frac{1}{Q_{1} Q_{2} x} \arctan \left(\frac{z x}{1-z \tau}\right), \quad x=\sqrt{1-\tau^{2}}, \\
z & =\frac{Q_{1} Q_{2}}{4 m_{\mu}^{2}}\left(1-\sigma_{1}^{E}\right)\left(1-\sigma_{2}^{E}\right), \quad \sigma_{i}^{E}=\sqrt{1+\frac{4 m_{\mu}^{2}}{Q_{i}^{2}}}
\end{aligned}
$$

The sum over the infinite tower of axial vector mesons corresponds to replacing the products of two form factors $A$ and the denominator of the axial vector meson propagator by one double-integral expression involving the bulk-to-bulk propagator as discussed in Sec. IV, e.g.,

$$
\sum_{n=1}^{\infty} \frac{A_{(n)}\left(Q_{1}^{2}, Q_{2}^{2}\right) A_{(n)}\left(Q_{3}^{2}, 0\right)}{Q_{3}^{2}+\left(M_{n}^{A}\right)^{2}}=\frac{4 g_{5}^{2}}{Q_{1}^{2} Q_{3}^{2}} \int_{0}^{z_{0}} d z\left[\frac{d}{d z} \mathcal{J}\left(Q_{1}, z\right)\right] \mathcal{J}\left(Q_{2}, z\right) \int_{0}^{z_{0}} d z^{\prime}\left[\frac{d}{d z^{\prime}} \mathcal{J}\left(Q_{3}, z^{\prime}\right)\right] G^{A}\left(Q_{3} ; z, z^{\prime}\right),
$$

which in longitudinal contributions reduces to

$$
\sum_{n=1}^{\infty} \frac{A_{(n)}\left(Q_{1}^{2}, Q_{2}^{2}\right) A_{(n)}\left(Q_{3}^{2}, 0\right)}{\left(M_{n}^{A}\right)^{2}}=\frac{4 g_{5}^{2}}{Q_{1}^{2} Q_{3}^{2}} \int_{0}^{z_{0}} d z\left[\frac{d}{d z} \mathcal{J}\left(Q_{1}, z\right)\right] \mathcal{J}\left(Q_{2}, z\right) \int_{0}^{z_{0}} d z^{\prime}\left[\frac{d}{d z^{\prime}} \mathcal{J}\left(Q_{3}, z^{\prime}\right)\right] G^{A}\left(0 ; z, z^{\prime}\right)
$$

As mentioned above, we have checked that our results agree upon integration with those obtained in an alternative derivation using the formalism of Refs. [20,63]. The latter agree with those given in Appendix C of Ref. [65].

[1] F. Jegerlehner, The anomalous magnetic moment of the muon, Springer Tracts Mod. Phys. 274, 1 (2017).

[2] F. Jegerlehner, Muon $g-2$ theory: The hadronic part, EPJ Web Conf. 166, 00022 (2018).
[3] M. Davier, A. Hoecker, B. Malaescu, and Z. Zhang, A new evaluation of the hadronic vacuum polarisation contributions to the muon anomalous magnetic moment and to $\alpha\left(m_{Z}^{2}\right)$, Eur. Phys. J. C 80, 241 (2020). 
[4] A. Keshavarzi, D. Nomura, and T. Teubner, $g-2$ of charged leptons, $\alpha\left(M_{Z}^{2}\right)$, and the hyperfine splitting of muonium, Phys. Rev. D 101, 014029 (2020).

[5] M. Abe et al., A new approach for measuring the muon anomalous magnetic moment and electric dipole moment, Prog. Theor. Exp. Phys. 2019, 053 C02 (2019).

[6] J. Prades, E. de Rafael, and A. Vainshtein, The hadronic light-by-light scattering contribution to the muon and electron anomalous magnetic moments, Adv. Ser. Dir. High Energy Phys. 20, 303 (2009).

[7] A. Kurz, T. Liu, P. Marquard, and M. Steinhauser, Hadronic contribution to the muon anomalous magnetic moment to next-to-next-to-leading order, Phys. Lett. B 734, 144 (2014).

[8] G. Colangelo, M. Hoferichter, A. Nyffeler, M. Passera, and P. Stoffer, Remarks on higher-order hadronic corrections to the muon $g-2$, Phys. Lett. B 735, 90 (2014).

[9] M. Hoferichter, B.-L. Hoid, B. Kubis, S. Leupold, and S. P. Schneider, Dispersion relation for hadronic light-by-light scattering: pion pole, J. High Energy Phys. 10 (2018) 141.

[10] I. Danilkin, C. F. Redmer, and M. Vanderhaeghen, The hadronic light-by-light contribution to the muon's anomalous magnetic moment, Prog. Part. Nucl. Phys. 107, 20 (2019).

[11] D. Giusti, V. Lubicz, G. Martinelli, F. Sanfilippo, and S. Simula, Electromagnetic and strong isospin-breaking corrections to the muon $g-2$ from Lattice QCD + QED, Phys. Rev. D 99, 114502 (2019).

[12] C. Davies et al. (Fermilab Lattice, LATTICE-HPQCD, MILC Collaborations), Hadronic-vacuum-polarization contribution to the muon's anomalous magnetic moment from four-flavor lattice QCD, Phys. Rev. D 101, 034512 (2020).

[13] A. Gérardin, H. B. Meyer, and A. Nyffeler, Lattice calculation of the pion transition form factor with $N_{f}=2+1$ Wilson quarks, Phys. Rev. D 100, 034520 (2019).

[14] A. Gérardin, M. Cé, G. von Hippel, B. Hörz, H. B. Meyer, D. Mohler, K. Ottnad, J. Wilhelm, and H. Wittig, The leading hadronic contribution to $(g-2)_{\mu}$ from lattice QCD with $N_{\mathrm{f}}=2+1$ flavours of $\mathrm{O}(a)$ improved Wilson quarks, Phys. Rev. D 100, 014510 (2019).

[15] T. Blum, N. Christ, M. Hayakawa, T. Izubuchi, L. Jin, C. Jung, and C. Lehner, Hadronic Light-by-Light Scattering Contribution to the Muon Anomalous Magnetic Moment from Lattice QCD, Phys. Rev. Lett. 124, 132002 (2020).

[16] S. Borsanyi et al., Leading-order hadronic vacuum polarization contribution to the muon magnetic moment from lattice QCD, arXiv:2002.12347.

[17] T. Aoyama, T. Kinoshita, and M. Nio, Revised and improved value of the QED tenth-order electron anomalous magnetic moment, Phys. Rev. D 97, 036001 (2018).

[18] A. Czarnecki, W. J. Marciano, and A. Vainshtein, Refinements in electroweak contributions to the muon anomalous magnetic moment, Phys. Rev. D 67, 073006 (2003); 73, 119901 (2006);

[19] C. Gnendiger, D. Stöckinger, and H. Stöckinger-Kim, The electroweak contributions to $(g-2)_{\mu}$ after the Higgs boson mass measurement, Phys. Rev. D 88, 053005 (2013).

[20] G. Colangelo, M. Hoferichter, M. Procura, and P. Stoffer, Dispersion relation for hadronic light-by-light scattering: Theoretical foundations, J. High Energy Phys. 09 (2015) 074.
[21] J. Leutgeb, J. Mager, and A. Rebhan, Pseudoscalar transition form factors and the hadronic light-by-light contribution to the anomalous magnetic moment of the muon from holographic QCD, Phys. Rev. D 100, 094038 (2019).

[22] H. R. Grigoryan and A. V. Radyushkin, Pion form-factor in chiral limit of hard-wall AdS/QCD model, Phys. Rev. D 76, 115007 (2007).

[23] H. R. Grigoryan and A. V. Radyushkin, Anomalous form factor of the neutral pion in extended AdS/QCD model with Chern-Simons term, Phys. Rev. D 77, 115024 (2008).

[24] H. R. Grigoryan and A. V. Radyushkin, Pion in the holographic model with 5D Yang-Mills fields, Phys. Rev. D 78, 115008 (2008).

[25] D. K. Hong and D. Kim, Pseudo scalar contributions to light-by-light correction of muon $g-2$ in AdS/QCD, Phys. Lett. B 680, 480 (2009).

[26] L. Cappiello, O. Cata, and G. D'Ambrosio, The hadronic light by light contribution to the $(g-2)_{\mu}$ with holographic models of QCD, Phys. Rev. D 83, 093006 (2011).

[27] J. Erlich, E. Katz, D. T. Son, and M. A. Stephanov, QCD and a Holographic Model of Hadrons, Phys. Rev. Lett. 95, 261602 (2005).

[28] L. Da Rold and A. Pomarol, Chiral symmetry breaking from five-dimensional spaces, Nucl. Phys. B721, 79 (2005).

[29] J. Hirn and V. Sanz, Interpolating between low and high energy QCD via a 5-D Yang-Mills model, J. High Energy Phys. 12 (2005) 030.

[30] K. Melnikov and A. Vainshtein, Hadronic light-by-light scattering contribution to the muon anomalous magnetic moment revisited, Phys. Rev. D 70, 113006 (2004).

[31] P. Achard et al. (L3 Collaboration), $f_{1}(1285)$ formation in two-photon collisions at LEP, Phys. Lett. B 526, 269 (2002).

[32] P. Achard et al. (L3 Collaboration), Study of resonance formation in the mass region 1400-1500 MeV through the reaction $\gamma \gamma \rightarrow K_{S}^{0} K^{ \pm} \pi^{\mp}$, J. High Energy Phys. 03 (2007) 018.

[33] V. Pauk and M. Vanderhaeghen, Single meson contributions to the muon's anomalous magnetic moment, Eur. Phys. J. C 74, 3008 (2014).

[34] G. Colangelo, F. Hagelstein, M. Hoferichter, L. Laub, and P. Stoffer, Short-distance constraints on hadronic light-by-light scattering in the anomalous magnetic moment of the muon, Phys. Rev. D 101, 051501 (2020); Longitudinal shortdistance constraints for the hadronic light-by-light contribution to $(g-2)_{\mu}$ with large- $N_{c}$ Regge models, J. High Energy Phys. 03 (2020) 101.

[35] K. Melnikov and A. Vainshtein, On dispersion relations and hadronic light-by-light scattering contribution to the muon anomalous magnetic moment, arXiv:1911.05874.

[36] J. M. Maldacena, The Large $N$ limit of superconformal field theories and supergravity, Int. J. Theor. Phys. 38, 1113 (1999).

[37] E. Witten, Anti-de Sitter space, thermal phase transition, and confinement in gauge theories, Adv. Theor. Math. Phys. 2, 505 (1998).

[38] T. Sakai and S. Sugimoto, Low energy hadron physics in holographic QCD, Prog. Theor. Phys. 113, 843 (2005).

[39] T. Sakai and S. Sugimoto, More on a holographic dual of QCD, Prog. Theor. Phys. 114, 1083 (2005). 
[40] A. Karch, E. Katz, D. T. Son, and M. A. Stephanov, Linear confinement and AdS/QCD, Phys. Rev. D 74, 015005 (2006).

[41] D. T. Son and N. Yamamoto, Holography and anomaly matching for resonances, arXiv:1010.0718.

[42] A. Vainshtein, Perturbative and nonperturbative renormalization of anomalous quark triangles, Phys. Lett. B 569, 187 (2003).

[43] M. Knecht, S. Peris, M. Perrottet, and E. de Rafael, New nonrenormalization theorems for anomalous three point functions, J. High Energy Phys. 03 (2004) 035.

[44] P. Colangelo, F. De Fazio, J. J. Sanz-Cillero, F. Giannuzzi, and S. Nicotri, Anomalous $A V^{*} V$ vertex function in the softwall holographic model of QCD, Phys. Rev. D 85, 035013 (2012).

[45] E. Witten, Current algebra theorems for the U(1) "Goldstone Boson”, Nucl. Phys. B156, 269 (1979).

[46] G. Veneziano, U(1) without instantons, Nucl. Phys. B159, 213 (1979).

[47] L. Bartolini, F. Bigazzi, S. Bolognesi, A. L. Cotrone, and A. Manenti, Theta dependence in Holographic QCD, J. High Energy Phys. 02 (2017) 029.

[48] J. Leutgeb and A. Rebhan, Witten-Veneziano mechanism and pseudoscalar glueball-meson mixing in holographic QCD, Phys. Rev. D 101, 014006 (2020).

[49] O. Aharony and D. Kutasov, Holographic duals of long open strings, Phys. Rev. D 78, 026005 (2008).

[50] K. Hashimoto, T. Hirayama, F.-L. Lin, and H.-U. Yee, Quark mass deformation of holographic massless QCD, J. High Energy Phys. 07 (2008) 089.

[51] F. Brünner and A. Rebhan, Constraints on the $\eta \eta^{\prime}$ decay rate of a scalar glueball from gauge/gravity duality, Phys. Rev. D 92, 121902 (2015).

[52] M. Tanabashi et al., Review of particle physics, Phys. Rev. D 98, 030001 (2018).

[53] H. J. Kwee and R. F. Lebed, Pion form-factors in holographic QCD, J. High Energy Phys. 01 (2008) 027.
[54] L. D. Landau, On the angular momentum of a system of two photons, Dokl. Akad. Nauk Ser. Fiz. 60, 207 (1948).C.-N. Yang, Selection rules for the dematerialization of a particle into two photons, Phys. Rev. 77, 242 (1950).

[55] P. Roig and P. Sanchez-Puertas, Axial-vector exchange contribution to the hadronic light-by-light piece of the muon anomalous magnetic moment, Phys. Rev. D 101, 074019 (2020).

[56] G. A. Schuler, F. A. Berends, and R. van Gulik, Meson photon transition form-factors and resonance cross-sections in $e^{+} e^{-}$collisions, Nucl. Phys. B523, 423 (1998).

[57] V. Pascalutsa, V. Pauk, and M. Vanderhaeghen, Light-bylight scattering sum rules constraining meson transition form factors, Phys. Rev. D 85, 116001 (2012).

[58] R. Aaij et al. (LHCb Collaboration), Observation of $\bar{B}_{(s)} \rightarrow$ $J / \psi f_{1}(1285)$ Decays and Measurement of the $f_{1}(1285)$ Mixing Angle, Phys. Rev. Lett. 112, 091802 (2014).

[59] J. J. Dudek, R. G. Edwards, B. Joo, M. J. Peardon, D. G. Richards, and C. E. Thomas, Isoscalar meson spectroscopy from lattice QCD, Phys. Rev. D 83, 111502 (2011).

[60] H.-Y. Cheng, Revisiting axial-vector meson mixing, Phys. Lett. B 707, 116 (2012).

[61] M. Hoferichter and P. Stoffer, Asymptotic behavior of meson transition form factors, J. High Energy Phys. 05 (2020) 159.

[62] F. Jegerlehner and A. Nyffeler, The muon $g-2$, Phys. Rep. 477, 1 (2009).

[63] G. Colangelo, M. Hoferichter, M. Procura, and P. Stoffer, Dispersion relation for hadronic light-by-light scattering: Two-pion contributions, J. High Energy Phys. 04 (2017) 161.

[64] A. E. Dorokhov, A. P. Martynenko, F. A. Martynenko, A. E. Radzhabov, and A. S. Zhevlakov, The LbL contribution to the muon $g-2$ from the axial-vector mesons exchanges within the nonlocal quark model, EPJ Web Conf. 212, 05001 (2019).

[65] L. Cappiello, O. Cata, G. D’Ambrosio, D. Greynat, and A. Iyer, On axials and pseudoscalars in the hadronic light-bylight contribution to the muon $(g-2)$, arXiv:1912.02779. 